\title{
In Vivo Imaging of MMP-13 Activity Using A Specific Polymer-FRET Peptide Conjugate Detects Early Osteoarthritis And Inhibitor Efficacy
}

Aroa Duro-Castano ${ }^{\dagger}$, Ngee Han Lim ${ }^{\dagger}$, Isabelle Tranchant ${ }^{\dagger}$, Mehdi Amoura, Fabrice Beau, Heike Wieland, Otmar Kingler, Matthias Herrmann, Marc Narazé, Oliver Plettenburg, Vincent Dive*, María J. Vicent", Hideaki Nagase*

$\dagger$ These authors contributed equally to this work

Dr A. Duro-Castano, Dr M.J. Vicent.

Polymer Therapeutics Lab, Centro de Investigación Príncipe Felipe, Av. Eduardo Primo Yúfera 3, Valencia 46012, Spain. Email: mjvicent@cipf.es

Dr N.H. Lim, Dr. H. Nagase. Matrix Biology, The Kennedy Institute of Rheumatology, NDORMS, University of Oxford, Roosevelt Drive, Headington, Oxford, OX3 7FY, United Kingdom. Email: hideaki.nagase@kennedy.ox.ac.uk

Dr I. Tranchant, Mehdi Amoura Fabrice Beau, Dr. V. Dive. Service d'Ingénierie Moléculaire des Protéines (SIMOPRO), CEA, Labex LERMIT, Université Paris-Saclay, F-91191 Gif/Yvette, France. Email: vincent.dive@cea.fr

Dr H. Wieland, Dr O. Kingler, Dr. M. Herrmann, Dr. M. Nazaré, Dr. O. Plettenburg, Immunology \& Inflammation TA, Sanofi-Aventis Germany GmbH, Industrial Park, 65926 Frankfurt am Main, Germany.

Dr. M. Nazaré. Medicinal Chemistry Leibniz-Forschungsnstitut für Molekulare Pharmakologie (FMP), Campus Berlin-Buch, Robert-Roessle-Strasse 10, 13125, Berlin, Germany

Dr O. Plettenburg. Helmholtz Zentrum München, Deutsches Forschungszentrum für Gesundheit und Umwelt GmbH, Institute of Medicinal Chemistry, Ingolstädter Landstrasse 1, 85764, Neuherberg, Germany

Dr O. Pletenburg. Leibniz Universität Hannover, Schneiderberg 1B, 30167, Hannover, Germany

Keywords: Polymer Therapeutics, MMP-13 nanoprobe, osteoarthritis, early detection, live imaging 
Imaging earlier molecular changes in osteoarthritic (OA) joints is instrumental for the development of the disease-modifying drugs. To this end, a fluorescent resonance energy transfer-based peptide probe that is cleavable by matrix metalloproteinase 13 (MMP-13) has been developed. This protease degrades type II collagen, a major matrix component of cartilage. The probe exhibits high catalytic efficiency $\left(k_{c a t} / K_{M}=6.5 \times 10^{5} \mathrm{M}^{-1} \mathrm{sec}^{-1}\right)$ and high selectivity for MMP-13 over a set of 9 MMPs. To achieve optimal in vivo pharmacokinetics and tissue penetration, it has been further conjugated to a linear Lpolyglutamate chain of $30 \mathrm{kDa}$. The conjugate detects early biochemical events that occur in a surgically induced murine model of OA before major histological changes. The nanometric probe is suitable for the monitoring of the in vivo efficacy of an orally bioavailable MMP-13 inhibitor, which effectively blocks cartilage degradation during the development of OA. This new polymer-probe can therefore be a useful tool in detecting early OA, disease progression and in developing MMP-13-based disease-modifying drugs for OA.

\section{Introduction}

Osteoarthritis $(\mathrm{OA})$ is the most prevalent age-related degenerative joint disorder. About 27 million people suffer from OA in the U.S. alone and about $10 \%$ of male and $18 \%$ of females above 60 years of age are affected globally. ${ }^{[1,2]}$ With the expanding ageing population, it imposes a major socio-economic burden on society, but currently there is no effective disease-modifying treatment except joint replacement surgery.

OA is typified by a gradual loss of articular cartilage, deformation of bone at the margin and a low-grade synovitis, which eventually results in the impairment of joint function and disability. ${ }^{[3,4]}$ There are many possible causes of cartilage loss (e.g. 
mechanical load, injury, reactive oxygen species, aging, apoptosis, extracellular matrix disassembly, and inflammation) and many etiological factors (obesity, aging, and genetics) that contribute to OA, but the primary cause of cartilage destruction is elevated levels of active proteases that degrade extracellular matrix of articular cartilage. ${ }^{[3,4]}$ These proteases are primarily produced by cartilage itself and proteases such as matrix metalloproteinases (MMPs), adamalysin-like metalloproteinases with thrombospondin motifs (ADAMTSs), and cathepsins have been implicated. ${ }^{[5]}$ Among them, MMP-13 (collagenase 3) that cleaves cartilage type II collagen and ADAMTS-5 (aggrecanase 2) that degrades aggrecan core protein, are considered to be major targets for therapeutic development. ${ }^{[6-9]}$ Consequently, numerous inhibitors for those metalloproteinases have been designed and synthesized. ${ }^{[9-15]}$ Some potent inhibitors were evaluated in the clinics, but trials were discontinued.

One of the major limitations to the development of a disease-modifying therapy for $\mathrm{OA}$ is the lack of sufficient outcome-measures to demonstrate structural improvement of cartilage, particularly for diseases such as OA that progress very slowly. Conventional radiographic assessment of joint space narrowing identifies structural changes of OA characteristic of late-stage disease, whilst good advancement has been made with gadolinium-enhanced magnetic resonance imaging (MRI) for analysis of early OA. ${ }^{[16]}$ Should a disease-modifying therapy become available, its application would be most effective if given early in the course of the disease, before severe joint deterioration has occurred. In this regard, proteolytic degradation of aggrecan ${ }^{[17]}$ and collagen ${ }^{[18]}$ is seen in humans, suggesting that detecting their activities may be used in developing better diagnostic tools to identify early stages of the disease and in monitoring the efficacy of a proteinase-based disease-modifying therapy of OA. For this purpose non-invasive imaging to detect molecular changes before structural changes is required. 
We have previously reported an enzyme-activatable fluorescence resonance energy transfer (FRET) substrate for MMP-13 ${ }^{[19]}$ and we used it for the detection of MMP-13 activity in the mouse OA model induced surgically by destabilizing the medial meniscus (DMM), but because of the limited sensitivity of the probe, it could not discriminate the OA knees from the sham-operated until 8 weeks after surgery, when histological damage of the cartilage was apparent. ${ }^{[20]}$ Limited specificity of the probe toward MMP-13 (23 MMPs are expressed in human) in this study might be a limiting factor. In the present work, we have developed a more sensitive and highly selective FRET substrate towards MMP-13 using unnatural amino acids. The substrate probe was further conjugated to a $30 \mathrm{kDa}$ poly-L-glutamic acid (PGA) carrier, a biodegradable and biocompatible carrier which increased probe stability in circulation and enhanced its accumulation in the knee joints, resulting in higher sensitivity to detect MMP-13 activity. The PGA-MMP-13 conjugate was sensitive enough to detect early OA before structural damage of the cartilage. Using the newly developed probe we could monitor the efficacy of an orally administered MMP-13 inhibitor in vivo.

\section{Results}

\subsection{Construction of the FRET peptide P-18 to monitor MMP-13 activity}

Many synthetic peptide substrates of MMPs with natural amino acids have been reported, but only a few of them exhibited a strict selectivity when tested on a large panel of MMPs, thus limiting their use for the in vivo imaging of a particular MMP. This lack of selectivity is due to the structural homology between the catalytic domains of the 23 MMPs. ${ }^{[21]}$ To overcome this hurdle, we used unusual amino acids to better exploit subtle differences between MMP catalytic domains. ${ }^{[22]}$ As MMP-13 has an $\mathrm{S}_{1}$ ' subsite with a very deep channel, we developed peptides with the $\mathrm{P}_{1}$ ' position harboring unusual amino acids with 
very long side chains that are able to fill the MMP-13 $\mathrm{S}_{1}$ ' subsite. Starting from two coumarin FRET peptide substrates that are cleaved with high catalytic efficiency by MMP-13, eighteen analogues were synthesized by substituting the $\mathrm{P}_{1}$ ' position with diverse unusual amino acids. Various $\mathrm{P}_{1}$ ' modifications were cleaved with high catalytic efficiency $\left(k_{c a t} / K_{M}\right)$ by MMP-13 (Figure S1, Probes 1-17). In this series, the presence of bi-phenyl triazole side chain in $\mathrm{P}_{1}$ ' position generates a substrate (Probe $\mathrm{P}-17$ ), exhibiting the highest catalytic efficiency towards MMP-13 $\left(1.8 \times 10^{6} \mathrm{M}^{-1} \mathrm{sec}^{-1}\right)$.

These results led us to synthesize the probe $\mathbf{1 8}(\mathrm{P}-\mathbf{1 8})$ that contains a bi-phenyl triazole in $\mathrm{P}_{1}$ ' position and Cy5.5 as a near infrared (NIR) fluorescent group and an NIR quencher group QSY21. To avoid steric clash between MMP-13 active site and the bulky NIR and QSY21 groups, they were not attached directly to the N- nor C-terminus of the peptide, but through two short polyethylene glycol (PEG) moieties (Figure 1A). An alkyne group was introduced to permit further probe modification. These chemical modifications resulted in a 4-fold decrease of the MMP-13 catalytic efficiency $\left(1.8 \times 10^{6}\right.$ $\mathrm{M}^{-1} \mathrm{sec}^{-1}$ versus $0.46 \times 10^{6} \mathrm{M}^{-1} \mathrm{sec}^{-1}$ ), but more importantly P-18 still exhibits an excellent selectivity profile when tested on a panel of nine MMPs (Figure 1B), where only a weak activity was detected with MMP-2. P-18 was also assayed with cathepsins B and L, but these two proteases cleave P-18 only at a very low efficiency $\left(<10^{2} \mathrm{M}^{-1} \mathrm{sec}^{-1}\right)($ Figure S2).

\subsection{Poly-L-glutamic acid (PGA) as an effective carrier of imaging probes to the cartilage}

Pharmacokinetics of P-18 indicated that it disappeared from the circulation with a rapid phase of distribution (half-life $\left(\mathrm{t}_{1 / 2} \alpha\right)$ of $\sim 140 \mathrm{~min}$ ) and a slower clearance phase (halflife $\left(\mathrm{t}_{1 / 2} \beta\right)$ of $\sim 600 \mathrm{~min}$ ) after intravenous (i.v.) injection (Figure 2 ). To improve biodistribution of P-18 and the quantity delivered to the articular joints, we considered its 
conjugation to a biodegradable polymer carrier. We chose PGA polymer of $30 \mathrm{kDa}$, as it has been a good candidate for the delivery of drugs and imaging probes, ${ }^{[23-25]}$ and we first investigated its suitability by monitoring the tissue distribution of PGA-Cy5.5. ${ }^{[26]}$ Our initial injection of PGA-Cy5.5 to mice showed higher levels of fluorescence compared than those with Cy5.5 alone in various organs including knee joints (Figure 2C). The mean fluorescence intensity in arbitrary units (MFI, a.u.) of PGA-Cy5.5 in knee joints at one day after i.v. injection, suggests that a PGA-conjugated probe would accumulate more efficiently in cartilage than the probe alone. Histological evaluation of the cartilage samples revealed co-localization of PGA-Cy5.5 with perlecan, a protein marker of cartilage defect (Figure 2D).

Based on these observations we conjugated P-18 to PGAcoEG(6) $\mathrm{N}_{3}$ by the copper(I)-catalyzed alkyne-azide cycloaddition "click reaction", [27] and purified the resulting nanometric probe (Figure S3, and Tables S1 and S2). The catalytic efficiency of P-18 was retained and even increased upon conjugation $\left(k_{c a t} / K_{m}\right.$ values of $46 \times 10^{4} \mathrm{M}^{-}$ ${ }^{1} \mathrm{sec}^{-1}$ for free P-18 versus $65 \times 10^{4} \mathrm{M}^{-1} \mathrm{sec}^{-1}$ for the conjugate PGA-P-18) (Figure 1B). The resulting conjugate maintained its selectivity toward MMP-13, even when the cleavage assays were performed in diluted plasma (Figure S4). It is noteworthy to observe full stability of PGA-P-18 in diluted plasma. However, pharmacokinetics of PGA-P-18 unexpectedly revealed much shorter biodistribution and clearance half-life $\left(\mathrm{t}_{1 / 2}\right.$ $\alpha$ of $\sim 80 \mathrm{~min}$ and $\mathrm{t}_{1 / 2} \beta$ of $\sim 600 \mathrm{~min}$ ) than P-18 (Figure $\mathbf{2 A}$ and $\mathbf{2 B}$ ). Absence of fluorescence increase in blood samples suggests that both probes remain intact in circulation.

\subsection{In vivo imaging of MMP-13 activity by the PGA-P-18 conjugate}

To compare the abilities of P-18 alone and the PGA-P-18 conjugate to detect MMP-13 activity in the OA knees, each probe was i.v. injected at equivalent doses through the tail 
vein at 6 weeks after DMM surgery, where only minimal cartilage structural damage was observed by capturing the emission signals at greater than $700 \mathrm{~nm}$ (Figure 3). Time course measurement of the signals from P-18 in the operated joints indicated the highest peak was at $8 \mathrm{~h}$, when there is only a very low amount of P-18 in blood after injection of the probe, with the MFI value of $\sim 1,190$. However, there were no significant differences with the controls (contra-lateral or sham-operated knees) (Figure 3A). The signals then declined gradually to the levels similar to the control knees at $48 \mathrm{~h}$. This observation may suggest a non-specific cleavage of P-18 in joints, followed by a probe clearance. By contrast, with PGA-P-18, significantly elevated MFI values were detected in DMM mice with a signal peaked earlier and increased up to $48 \mathrm{~h}$ post conjugate injection of the probe (Figure 3B). As compared with P-18, these differences indicate a better retention of PGAP-18 in joint that may favor specific cleavage by MMP-13 (see below). These led to a clear distinction between signals in DMM mice and those in controls (contra-lateral or sham-operated knees). The specific cleavage of the PGA-P-18 probe by MMP-13 was demonstrated by the almost complete signal inhibition after oral administration of MMP13 inhibitor A4727 (Figure 3C). A4727 is an orally bioavailable MMP-13 inhibitor developed by Sanofi (see Figure 5A for the chemical structure), which has been designed based on the pyrimidine dicarboxamide inhibitors originally described by Engel et al. ${ }^{[28]}$ The inhibitor was highly selective for MMP-13 with nanomolar $K_{i}$ value $(8 \mathrm{nM})$, with inhibition constants greater than $10 \mu \mathrm{M}$ against MMPs-1, -2, -3, -8, -9 and -14 are (Table 1). ADAMTS-4 and ADAMTS-5 were not inhibited by A4727 at least at $10 \mu \mathrm{M}$.

At 8 weeks post-surgery, where there is already cartilage damage, $\mathrm{P}-\mathbf{1 8}$ was able to discriminate between both controls and DMM-damaged knees (with the MFI value of 1,300) (Figure S5A). This suggests a higher level of MMP-13 expression at 8 weeks post-surgery favoring the possibility to observe a specific cleavage of P-18 by MMP-13, 
but again the fluorescence signal peaked at $8 \mathrm{~h}$ post-injection and then declined reaching a value similar between DMM and controls. With PGA-P-18 (Figure S5B), a clear distinction was observed between DMM and controls and this difference extended up to $150 \mathrm{~h}$ post-injection.

\subsection{Early OA detection in mice by the PGA-P-18 conjugate}

The DMM mice develop OA slowly in a time-dependent manner. In our experimental model, the mice showed little histological cartilage damage at 4 weeks, and only very minimal structural damage was observed at 6 weeks after surgery, which gradually progressed to more damaged cartilage in time (see Figure 1 in Ref. ${ }^{[20]}$ ). To determine the earliest time point that we can detect by PGA-P-18, we imaged the knee joints every two weeks after DMM surgery and images were captured two days after i.v. injection of the probe, Representative images are shown in Figure 4A. Quantification and statistical analyses of the fluorescence signals revealed little increase at 2 weeks after DMM surgery, and higher signals were detected at 4 weeks (Figure 4B). We could not detect statistically significant signals at 4 weeks, due unfortunately to large signal variations in the DMM group. The earliest time point at which we could detect significant differences was at 6 weeks; MFI signals were $\sim 1,500$ for operated knees, compared with 920 for contra-lateral and $~ 970$ for sham-operated knees, giving a specific signal and noise ratio of about 1.5. Histological signs of damage in DMM joints appeared at 8 weeks indicated that collagenolytic activity was significantly elevated at an early stage in the development of the OA model, before major histological changes of cartilage occurred.

\subsection{Monitoring the efficacy of MMP-13 inhibitor A4727 in the OA model}

PGA-P-18 was then tested for efficacy of the MMP-13 inhibitor A4727 in real time in vivo and whether it prevents degradation of cartilage in OA knees as a potential OA-modifying drug. Mice were subjected to DMM surgery on their right knees, and two 
weeks later they were orally treated with A4727 in the dose of $0.3 \mathrm{mg} / \mathrm{kg}$ or $30 \mathrm{mg} / \mathrm{kg}$ daily. Then, knee joints were measured with PGA-P-18 every two weeks starting at 6 weeks after surgery and up to 12 weeks. As shown in Figure 5B, MMP-13 activity was completely blocked by oral administration of A4727 at the dose of $30 \mathrm{mg} / \mathrm{kg}$ A4727 and partially by $0.3 \mathrm{mg} / \mathrm{kg}$. Histological analysis of the knees at 12 weeks with Safranin O staining for proteoglycans indicated that the degree of cartilage protection correlated well with the blockade of the MMP-13 activity (Figures 5C and 5D). The integrity of cartilage structure of the mice treated with $30 \mathrm{mg} / \mathrm{kg}$ of A4727 appeared essentially normal, compared with those of untreated DMM mice and DMM mice treated with lower dose of A4727. However, there was some loss of aggrecan in the surface area of cartilage in these mice. These results suggest that aggrecan-degrading proteases are locally active, but the inhibition of MMP-13 activity was sufficient to protect the cartilage from substantial structural damage.

\section{Discussion}

One of the major hurdles to the discovery of disease-modifying drugs for OA is the lack of suitable methods to readout the efficacy of the drugs in humans, as the progression of the disease is very slow. MMP-13 is an enzyme that cleaves a major structural macromolecule type II collagen in cartilage and other matrix components. ${ }^{[29]}$ Thus, inhibitors for MMP-13 are considered to be therapeutic candidates. ${ }^{[30]}$ In this study, we have synthesized a highly selective and sensitive non-invasive in vivo imaging probe to detect MMP-13 activity. The probe PGA-P-18 consists of a NIR-FRET peptide and a 30 $\mathrm{kDa}$ poly-L-glutamic acid polymer that allows the probe to rapidly accumulate in the cartilage and be retained. We have demonstrated its activation in the mouse model of OA 
before major structural joint damage takes place, and its ability to monitor in vivo efficacy of an orally administered MMP-13 inhibitor as a potential disease-modifying drug of OA. NIR fluorescent probes are used for in vivo optical imaging. ${ }^{[31]}$ A proteaseselective peptide flanked by a NIR fluorescent donor and a NIR fluorescent acceptor can sense the activity of proteases, ${ }^{[32,33]}$ and a number of them have been generated. ${ }^{[34]}$ Such probes are highly fluorescent after cleavage of the peptide bond by target proteinases. Previously, Lee et al. ${ }^{[35]}$ synthesized an activatable fluorescent probe to detect the MMP13 activity in the rat OA model induced by DMM. This was based on the peptide sequence reported by Deng et al. ${ }^{[36]}$ but the selectivity profile of the probe toward many MMPs was not characterized as well as for PGA-P-18. It could be cleaved by MMP-9 and other MMPs that are thought to be elevated in OA. ${ }^{[37]}$ Another significant difference between their work ${ }^{[35]}$ and the present study was that they injected the probe directly into the knee joints. In our study PGA-P-18 was administered systemically and detected specifically MMP-13 activity in the affected joints. More recently, Leahy et al. ${ }^{[38]}$ detected increased MMP activity at 4 weeks after DMM surgery in mice. While their study is interesting, they used the general MMP probe MMPSense 680, which does not discriminate a single MMP activity, avoiding to conclude about the identity of the MMP(s) cleaving this probe. We aimed to detect specifically MMP-13 activity and monitor the efficacy of MMP-13 inhibitors with the intention of developing future OA drugs that protect the joints from destruction. To fill this goal, a structure-based design has been used leading us to select unusual amino acids in the $\mathrm{P}_{1}$ ' position of peptide substrate. This strategy led us to identify first P-18 and then developed PGA-P-18 probes that exhibit the best MMP-13 selectivity profile reported to date when assayed on nine MMPs, while retaining very catalytic efficiency towards MMP-13. Aside selectivity profile, high catalytic efficiency is also critical to favor specific probe cleavage by the targeted protease, in a context of 
inflammatory disease characterized by the expression of several proteases. Establishing peptide substrate selectivity towards a particular MMP in vivo has been hampered in many studies by the lack of potent and highly selective inhibitor of MMPs. The inhibitor used in this study is an exception in the field, as it exhibits nanomolar potency toward MMP13, but micromolar one when tested on other MMPs. Thus, A4727 inhibitor was the ideal tool to demonstrate in vivo that PGA-P-18 probe is mostly cleaved by MMP-13, in line with its in vitro specificity profile. The presence of a by-phenyl triazol side chain in P-18 and PGA-P-18 probes probably protects probe degradation by proteases not belonging to the MMP family. However, as compared to P-18, our data with PGA-P-18 suggest that fast accumulation and long retention of the probe in joint knee, due to PGA biodistribution property, is also playing a key role to insure specific probe cleavage by MMP-13. Indeed, P-18 failed to detect MMP13 activity at earlier stages OA of joints. Through its conjugation with PGA, we could observe a statistically significant increase in MMP-13 activity at 6 weeks after DMM surgery, when cartilage presents minimal degradation without observable histological changes. Pharmacokinetic studies indicated that both P18 and PGA-P-18 remained intact in blood and were mostly hydrolyzed in cartilage, but P-18 was not retained in cartilage. Polymer conjugation is known to have beneficial effects on tissue biodistribution. ${ }^{[26]}$ Various sizes of PGA have been explored for their ability to penetrate cartilage matrix that restricts materials by size, charge, and molecular configuration. It is estimated that the average pore diameter is around 11-14 nm (in cartilage matrix), ${ }^{[39]}$ thus molecular masses are also determining factors. Polymers of $35 \mathrm{kDa}$ have been reported to be retained in articular cartilage up to 10 -fold more than smaller polymers $(6-10 \mathrm{kDa})$ and bigger polymers $(>200 \mathrm{kDa})$ were not retained at all. ${ }^{[40]}$ Thus, it is clear that the PGA size (> $14 \mathrm{~nm})$, molecular mass $(30 \mathrm{kDa})$, and negative 
charge used in this study have a strong influence on penetration through synovial membranes and accumulation in hyaline cartilage.

Blocking MMP-13 by oral administration of the MMP-13 inhibitor A4727 to the mice resulted in an almost complete suppression of cartilage matrix degradation even at 12 weeks after surgery. This observation is similar to that reported for MMP-13-null mice, which were subjected to the same surgical model. ${ }^{[7]}$ However, as in the case of MMP-13null mice, the focal degradation of aggrecan was noticeable at 12 weeks after surgery. This indicates that aggrecanase activity derived from ADAMTS metalloproteinases is not suppressed by A4727. Nevertheless, areas of aggrecan degradation observed were very limited, suggesting only a partial activation of aggrecanases in the MMP-13 inhibitortreated mice. This is probably due to the protection of the overall structure of cartilage by blocking collagenolysis, indicating that collagen degradation of cartilage is a crucial part of OA progression, and that the cleavage of type II collagen and perhaps other components of extracellular matrix such as perlecan, tenascin $\mathrm{C}$ and fibronectin by MMP13 may be involved in OA. ${ }^{[29]}$

\section{Conclusion}

Currently there are a few molecular probes available to image a specific protease activities in vivo in articular joints ${ }^{[33-38]}$, but as previously pointed out, they have limitations and several parameters need to be optimized to foster their suitable application to image OA progression. Furthermore, multiple proteases most likely act in different combination at different progressive stages of OA. To block these pathological processes with protease inhibitors as a potential therapy, we need to profile protease activities in the cartilage at different stages of the disease. Molecular imaging technology with activitybased probes are essential for such information. In this regard, we report here the 
development of the first macromolecular and biodegradable polypeptidic in vivo specific MMP-13 cleavable probe. PGA-P-18 has demonstrated a remarkable accumulation within the pathological site of action after systemic i.v. administration, possibly due to the tissue specificity provided by polymer conjugation. The nanometric probe presented in this work displays an unique specificity to sense MMP-13 activity in vivo, with a full blockade of the reporter probe in the presence of a very potent and selective MMP-13 inhibitor. This contrast to previous works where the MMP reporter is still cleaved (even up to $40 \%$ extend) under the treatment of a very potent MMP inhibitor. ${ }^{[41]}$ As demonstrated in this study, the polymeric probe allows non-invasive quantitative assessment of target expression in diseased tissue (diagnosis) and monitoring of disease progression (staging). Overall, it represents a powerful tool for understanding the molecular processes, and concretely, the role of enzymes during the formation and progression of diseases such OA. Besides, in the clinical setting it is likely to be suited in measuring the elevated MMP-13 activity in hand OA joints. The probe may be also useful in understanding the molecular processes and the role of MMP-13 activities in other chronic diseases such as atherosclorosis and various types of cancer.

\section{Experimental Section}

FRET peptide (P-18) synthesis. The P-18 peptide sequence was synthesized on NovaTagTM resin (Novabiochem) using a standard Fmoc methodology. The incorporation of QSY21 quencher, formation of the biphenyl triazole side chain through 1,3 dipolar cycloaddition as well as conjugation of the hex-5-ynoyl-L-lysine motif in Cterminal were successively performed on solid support. After cleavage from the support and RP-HPLC purification, the NHS- activated 6SIDCC-Cy5.5 was conjugated in solution. P18 was purified by RP-HPLC (7\% yield), characterized using appropriate 
analytical methods and stored at $-20^{\circ} \mathrm{C}$ away from light (see Supplementary Material for synthetic details).

Poly-L-glutamic acid (PGA)-P-18 conjugate synthesis. One eq. of the respective copolymer PGAcoEG(6)N $\mathrm{N}_{3}$ sodium salt (see Supplementary Materials for details) was used. The corresponding amount of P-18 measured spectrophotometrically was added to the mixture in the presence of 1 eq. of $\mathrm{CuSO}_{4}$, and the final product was purified determined by ${ }^{1} \mathrm{H}-\mathrm{NMR}$. Probe content was estimated also by spectroscopically according to the fluorescence of Cy5.5 $\left(\lambda_{\mathrm{ex}}=595 \mathrm{~nm}, \lambda_{\mathrm{em}}=680 \mathrm{~nm}\right)$. PGA-P-18 Yield were: $60 \%$ CE $75 \%$; mol probe to GAU $1.5 \%$ (2.3 eq. per polymer chain, according to fluorescence and confirmed by $\left.{ }^{1} \mathrm{H}-\mathrm{NMR}\right), 20.74 \mathrm{wt} \%, \mathrm{MW}=39.0 \mathrm{kDa}$.

Synthesis of MMP-13 inhibitor A4727. Pyrimidine-4,6-dicarboxylic acid 4 - [(2,3dihydro-benzofuran-5-ylmethyl) -amide] 6- [4- (morpholine-4- carbonyl) -benzylamide (A4727) was synthesized in four steps. Complete synthesis is fully described in Supplementary Materials.

Enzyme kinetics Analysis. Enzyme inhibition assays were carried out in $50 \mathrm{mM}$ Tris/HCl buffer, $\mathrm{pH} 6.8,10 \mathrm{mM} \mathrm{CaCl}_{2}$, at $25^{\circ} \mathrm{C}$. The assays were performed in black 96-well plates (non-binding surface plates 3651; Corning Costar). Progress curves were monitored by recording the increase in fluorescence resulting from substrate cleavage (Coumarin probe: $\lambda_{\mathrm{ex}}=320 \mathrm{~nm}, \lambda_{\mathrm{em}}=405 \mathrm{~nm}$; cyanine probe: $\left.\lambda_{\mathrm{ex}}=665 \mathrm{~nm}, \lambda_{\mathrm{em}}=695 \mathrm{~nm}\right)$. The conditions of a typical experiment were $100 \mu \mathrm{L}$ of buffer, $1-5 \mathrm{nM}$ of MMP or cathepsins $(\mathrm{R} \& \mathrm{R}$ systems). See Supplementary Materials for details.

Mouse OA model studies. DMM surgery was performed on 10 week old male C57BL/6J mice (Harlan Laboratories, Blackthorn, Bicester, UK) according to Glasson et al. ${ }^{[42]}$ with the exception that isofluorane $\left(2.5 \%, \mathrm{O}_{2}\right)$ was used for anesthesia, instead of the injectable 
anesthetics. ${ }^{[20]}$ Sham surgery consisted of the capsulotomy, but the meniscotibial ligament was not cut. All experimental protocols were performed in compliance with the UK Animals (Scientific Procedures) Act 1986 regulations for the handling and use of laboratory animals (Home Office project license PPL no:30/3129). After DMM surgery mice knees were i.v. injected via tail veins with $150 \mu 1$ of $1 \mu \mathrm{M}$ P-18 or PGA-P-18 at 2 , 4, 6, 8 weeks, and increased fluorescence images were detected in mice under gaseous anesthesia $\left(2.5 \%\right.$ isofluorane and $\mathrm{O}_{2}$ ) using the Kodak In Vivo FX Pro (Carestream, Woodbridge, USA) with excitation at $630 \mathrm{~nm}$ and emissions at greater than $700 \mathrm{~nm}$ for 1 min. Fluorescence images were analyzed using the Carestream MI software (version 5.1, Carestream, Woodbridge, USA.). X-ray images were taken at the same time for coregistration of signal to the knee joint by exposing to X-rays for $20 \mathrm{sec}$. To determine the contribution of MMP-13 to the in vivo cleavage of PGA-P-18 at 8 weeks post DMM surgery, mice were treated with $30 \mathrm{mg} / \mathrm{kg}$ of A 4727 orally 1 day before and 4 hours before imaging with PGA-P-18.

Histology and scoring of cartilage damage. At 12 week after DMM surgery, mice were sacrificed by cervical dislocation and the knee joints were isolated, decalcified and embedded in paraffin as described (20). Every $20^{\text {th }}$ section was stained with Safranin O for scoring of cartilage damage. Scoring was performed by two different observers, blinded to the treatment groups, in accordance with the guidelines laid out in the OARSI histopathology initiative for small animals on a scale from 1 to $6 .{ }^{[43]}$

Statistical analysis. All statistical analysis were performed with the software Prism (version 6.0, Graphpad Software, La Jolla, USA) using 2-way analysis of variance (ANOVA) with Bonferonni's post tests, with $p$ values corrected for multiple comparisons. 


\section{Acknowledgements}

The authors thank Bryony Stott for providing the histology and for the blind scoring of the sections. Funding: The work was funded through the EU FP7 grant LIVIMODE awarded to a consortium including VD, HN, MJV and Sanofi-Aventis, Spanish Ministry grant SAF2016-80427-R to MJV and NIH grant AR40994 to HN. Partly co-funded by FEDER (PO FEDER Valencian Community - 2014 - 2020). Author contributions: AD$\mathrm{C}$ and MJV synthesized and characterized PGA, PGA-Cy5.5, PGAcoEG(6)N3 and PGAP-18. IT and VD designed, developed and characterized P-18 and screened PGA-P-18 as MMP substrates. NHL and HN conducted in vivo imaging and inhibitor study in the mouse OA model. HW, OK, MH, MN and OP synthesized and characterized MMP-13 inhibitor A-4727. VD, AD-C, MJV, NHL and HN drafted the manuscript. All authors read and approved the final version of the manuscript. Competing interests: There is no financial interests of any of the authors and therefore there is no conflict of interest. H.W., O.K. and M.H. are employees of Sanofi-Aventis Deutschland GmbH. Data and materials availability: Materials described may be obtained through an MTA.

\section{References}

1. M. B. Goldring, F. Berenbaum, Curr. Opin. Pharmacol. 2015, 22, 51-63.

2. R. F. Loeser, J. A. Collins, B. O. Diekman, Nat. Revs. Rheum. 2016, 12, 412-

420.

3. A. D. Woolf, B. Pfleger, Bull. World Health Organ. 2003, 81, 646-656.

4. C. Palazzo, C. Nguyen, M. M. Lefevre-Colau, F. Rannou, S. Poiraudeau, Ann. Phys. Rehabil. Med. 2016, 59, 134-138.

5. L. Troeberg, H. Nagase, Biochim Biophys Acta 2012, 1824, 133-145.

6. P. G. Mitchell, H. A. Magna, L. M. Reeves, L. L. Lopresti-Morrow, S. A.

Yocum, P. J. Rosner, K. F. Geoghegan, J. E. Hambor, J. Clin. Invest. 1996, 97, 761768 .

7. C. B. Little, A. Barai, D. Burkhardt, S. M. Smith, A. J. Fosang, Z. Werb, M.

Shah, E. W. Thompson, Arthritis Rheum. 2009, 60, 3723-3733.

8. S. S. Glasson, R. Askew, B. Sheppard, B. Carito, T. Blanchet, H. L. Ma, C. R. Flannery, D. Peluso, K. Kanki, Z. Yang, M. K. Majumdar, E. A. Morris, Nature 2005, 434, 644-648. 
9. J. Larkin, T. A. Lohr, L. Elefante, J. Shearin, R. Matico, J. L. Su, Y. Xue, F. Liu, C. Genell, R. E. Miller, P. B. Tran, A. M. Malfait, C. C. Maier, C. J. Matheny, Osteoarthritis Cartilage 2015, 23, 1254-1266.

10. D. Piecha, J. Weik, H. Kheil, G. Becher, A. Timmermann, A. Jaworski, M.

Burger, M. W. Hofmann, Inflamm. Res. 2010, 59, 379-389.

11. P. J. Ruminski, M. Massa, J. Strohbach, C. E. Hanau, M. Schmidt, J. A.

Scholten, T. R. Fletcher, B. C. Hamper, J. N. Carroll, H. S. Shieh, N. Caspers, B.

Collins, M. Grapperhaus, K. E. Palmquist, J. Collins, J. E. Baldus, J. Hitchcock, H. P.

Kleine, M. D. Rogers, J. McDonald, G. E. Munie, D. M. Messing, S. Portolan, L. O.

Whiteley, T. Sunyer, M. E. Schnute, J. Med. Chem. 2016, 59, 313-327.

12. N. G.Li, Z. H. Shi, Y. P. Tang, Z. J. Wang, S. L. Song, L. H. Qian, D. W.

Qian, J. A. Duan, Curr. Med. Chem. 2011, 18, 977-1001.

13. T. B. Durham, V. J. Klimkowski, C. J. Rito, J. Marimuthu, J. L.Toth, C. Liu, J.

D. Durbin, S. L. Stout, L. Adams, C. Swearingen, C. Lin, M. G. Chambers, K.

Thirunavukkarasu, M. R.Wiley, J. Med. Chem. 2014, 57, 10476-10485.

14. A. M. Gilbert, M. G. Bursavich, S. Lombardi, K. E. Georgiadis, E. Reifenberg, C. R. Flannery, E. A. Morris, Bioorg. Med. Chem. Lett. 2007, 17, 1189-1192.

15. H. Deng, H. O'Keefe, C. P. Davie, K. E. Lind, R. A. Acharya, G. J. Franklin, J. Larkin,R. Matico, M. Neeb, M. M. Thompson, T. Lohr, J. W. Gross, P. A. Centrella, G. K. O'Donovan, K. L.Bedard ,K. van Vloten, S. Mataruse, S. R. Skinner, S. L. Belyanskaya, T. Y. Carpenter, T. W. Shearer, M. A. Clark, J. W. Cuozzo, C. C. AricoMuendel, B. A. Morgan, J. Med. Chem. 2012, 55, 7061-7079.

16. a) S. Glyn-Jones, A. J. Palmer, R. Agricola, A. J. Price, T. L. Vincent, H. Weinans, A. J. Carr, Osteoarthritis. Lancet 2015, 386, 376-387. b) H.-Y. Hu, N.-H. Lim, H. P. Juretschke, D. Ding-Pfennigdorff, P. Florian, M. Kohlmann, A. Kandira, J. P. von 
Kries, J. Saas, K. A.Rudolphi, U. K. Wendt, H. Nagase, O. Plettenburg, M. Nazare, C. Schultz, Chemical Science, 2015, 6, 6265-6261.

17. M. W. Lark, E. K. Bayne, J. Flanagan, C. F. Harper, L. A. Hoerrner, N. I. Hutchinson, I. I. Singer, S. A. Donatelli, J. R.Weidner, H. R. Williams, R. A. Mumford,L. S. Lohmander, J.Clin.Invest. 1997, 100, 93-106.

18. W. Wu, R. C. Billinghurst, I. Pidoux, J. Antoniou, D. Zukor, M. Tanzer, A. R. Poole, Arthritis and Rheumatism 2002, 46, 2087-2094.

19. N. H. Lim, E. Meinjohanns, G. Bou-Gharios, L. L. Gompels, E. Nuti, A. Rossello, L. Devel, V. Dive, M. Meldal, H. Nagase, Arthritis Rheum. 2014, 66, 589598.

20. N.H. Lim, E. Meinjohanns, M. Meldal, G. Bou-Gharios, H. Nagase, Osteoarthritis Cartilage 2014, 22, 862- 868.

21. C. Tallant, A. Marrero, Gomis-Rüth, F. X. Biochim. Biophys. Acta 2010, 1803, $20-28$.

22. I. Tranchant, L. Vera, B. Czarny, M. Amoura, E. Cassar, F. Beau, E. A. Stura, V. Dive, Chem. Biol. 2014, 21, 408-413.

23. A. Eldar-Boock, K. Miller, J. Sanchis, R. Lupu, M. J. Vicent, R. Satchi-Fainaro, Biomaterials 2011, 32, 3862-3874.

24. I. Conejos-Sanchez, I. Cardoso, M. J. Saraiva, M. J. Vicent, J Control Release 2014, $178,95-100$.

25. L. V. Kiew, H. Y. Cheah, S. H. Voon, E. Gallon,J. Movellan, K. H. Ng, Alpugan, S., H. B. Lee, F. Dumoulin,M. J. Vicent, L. Y. Chung, Nanomedicine 2017, $13,1447-1458$.

26. A. Duro-Castano, R. M. England, D. Razola, E.Romero, M. Oteo-Vives, M. A. Morcillo, M. J.Vicent Mol Pharm 2015, 12, 3639-3649. 
27. M. Barz, A. Duro-Castano, M. Vicent, Polym. Chem. 2013, 4, 2981-2994.

28. C. K. Engel, B. Pirard, S. Schimanski, R. Kirsch, J. Habermann, O. Klingler, V.

Schlotte, K. U. Weithmann, K. U. Wendt, Chem.Biol. 2005, 12, 181-189.

29. K. Yamamoto, H. Okano,W. Miyagawa, R. Visse, Y. Shitomi, S. Santamaria, J.

Dudhia, L.Troeberg,D. K. Strickland, S. Hirohata, H. Nagase. Matrix Biol. 2016, 56, $57-73$.

30. X. W. Xie,R. Z. Wan, Z. P. Liu, Z. P. Chem. Med. Chem. 2017, 12, 1157-1168.

31. U. Mahmood, R. Weissleder, Mol. Cancer. Ther. 2003, 2, 489-496

32. C. H. Tung. Biopolymers 2004, 76, 391-403

33. W. Pham, Y. Choi, R. Weissleder, C. H. Tung. Bioconjug. Chem. 2004, 15, 1403-1407.

34. C. R. Drake, D. C. Miller, E. F. Jones, Curr Org Synth. 2011, 8, 498-520.

35. S. Lee, K. Park, S. Y. Lee, J. H. Ryu, J. W. Park, H. J. Ahn, I. C. Kwon, I. C. Youn, K. Kim, K. Choi. Bioconjug. Chem. 2008, 19, 1743-1747.

36. S. J. Deng, D. M. , Bickett, J. L. Mitchell, M. H. Lambert, R. K. Blackburn, H. L. Carter, J. Neugebauer, G. Pahel, M. P. Weiner, M. L. Moss. J. Biol. Chem. 2000, $275,31422-31427$.

37. L. C. Tetlow,D. J. Adlam, D.E. Woolley. Arthritis Rheum. 2001, 44, 585-594.

38. A. A. Leahy, S. A. Esfahani, A. T. Foote, C. K. Hui, R. S. Rainbow, D. S.

Nakamura, B. H. Tracey, U. Mahmood, L. Zeng, Arthritis Rheum. 2015, 67, 442-453.

39. D. Majda, A. Bhattarai, J. Riikonen, B. D. Napruszewska, M. Zimowska, A.

Michalik-Zym, J. Töyräs, V. -P. Lehto. Microporous and Mesoporous Materials, 2017, $241,238-245$.

40. B. Sterner, M. Harms, S. Woll, M. Weigandt, M. Windbergs, C. M. Lehr, Eur. J. Pharm. Biopharm. 2016, 101, 126-136. 
41. Bremer C, Tung CH, Weissleder R. Nat Med. 2001, 7, 743-8

42. S. S. Glasson, T. J. Blanchet, E. A. Morris. Osteoarthritis. Cartilage. 2007, 15, 1061-1069.

43. S. S. Glasson, M. G. Chambers, W. B. Van Den Berg, C. B. Little,

Osteoarthritis Cartilage 2010, 18 Suppl 3, S17-23.

44. E. O. Stejskal, J. E. Tanner,. J. Chem. Phys. 1965, 42, 288-292.

45. I. Conejos-Sanchez, A. Duro-Castano, A. Birke, M. Barz, M. Vicent. Polym.

Chem. 2013, 4, 3182-3186.

A

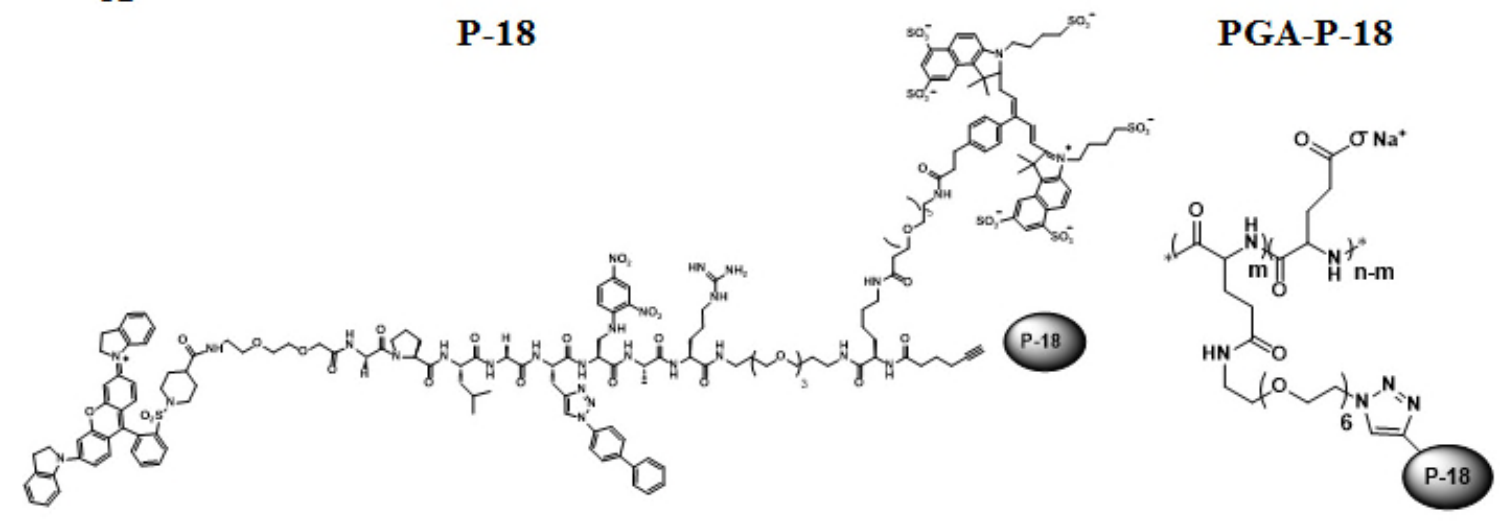

B

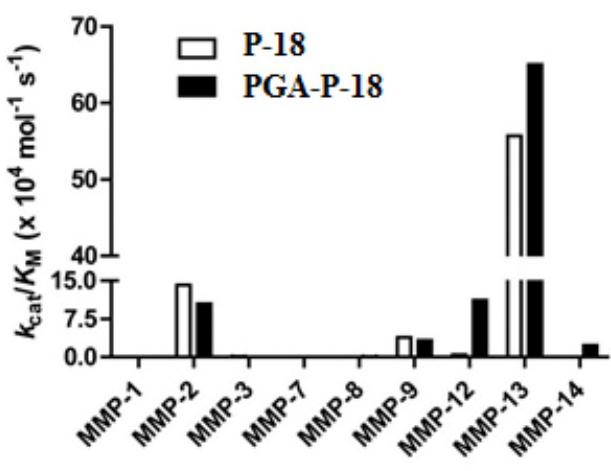

C

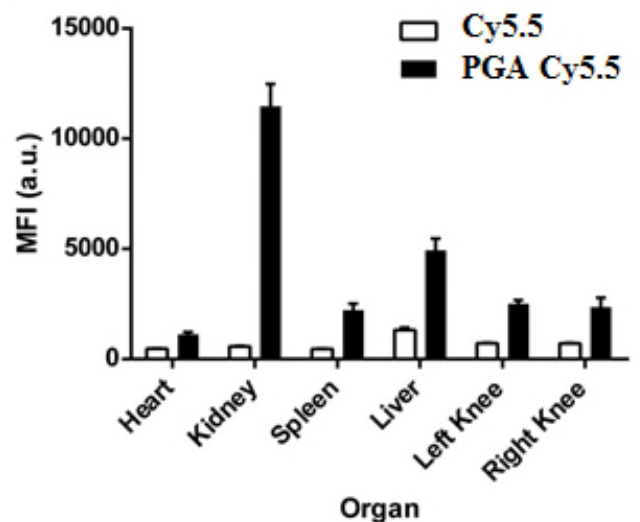

Figure. 1. Characterization of P-18 and PGA-P-18. (A) Chemical structures of P-18 and PGA-P-18. (B) In vitro specificity of cleavage of P-18 and PGA-P-18 by the different MMPs, expressed as $k_{\mathrm{cat}} / K_{\mathrm{M}}\left(\mathrm{M}^{-1} \mathrm{sec}^{-1}\right)$ determination in PBS buffer. (C) Distribution of PGA labeled with Cy5.5 1 day after i.v. injection, as determined by ex vivo fluorescence imaging of organs that are expressed as mean fluorescence intensity in arbitrary units (MFI, a.u.). Data is mean $\pm \mathrm{SD}, \mathrm{n}=3$. 


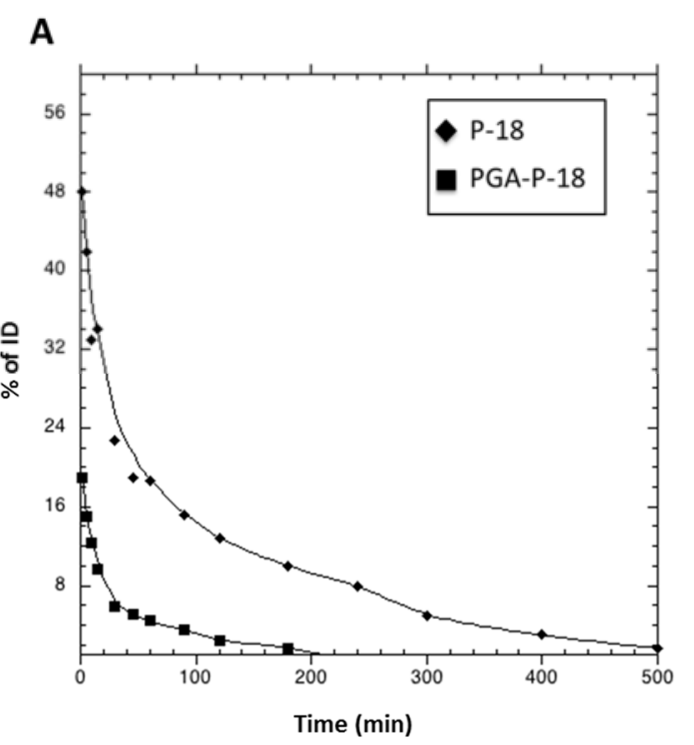

C

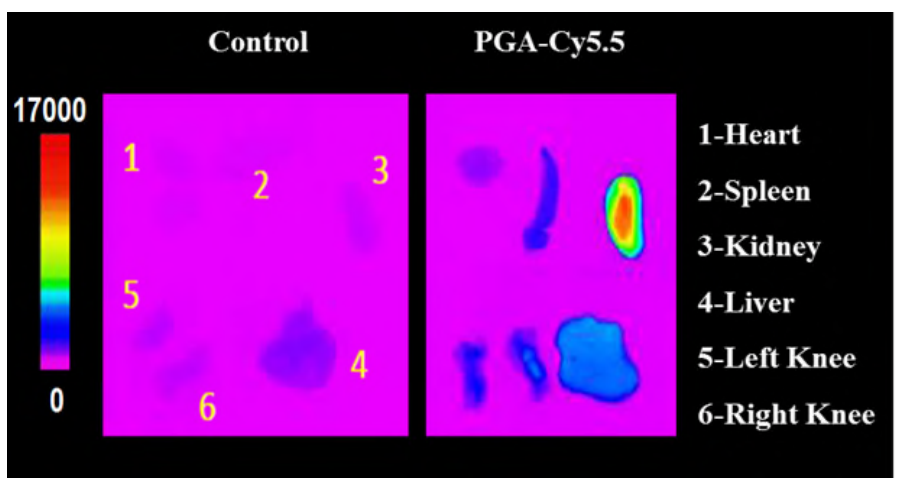

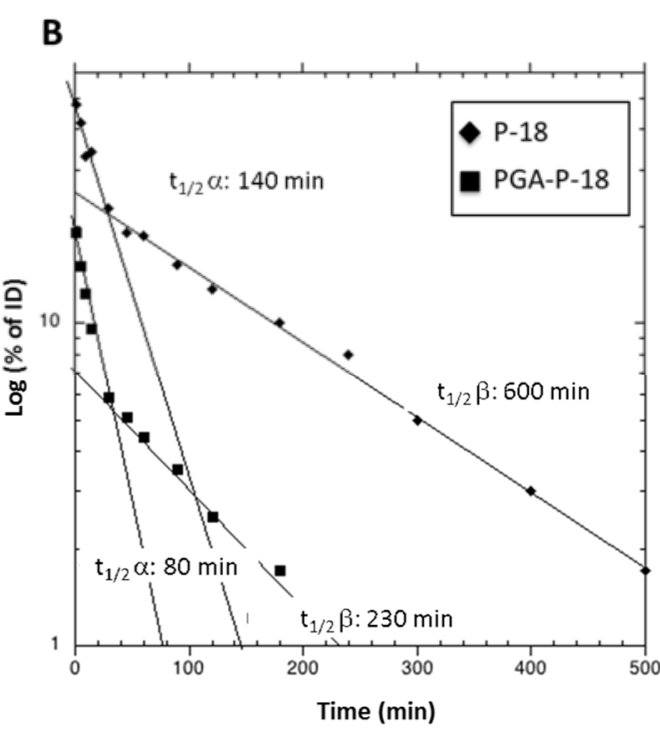

D

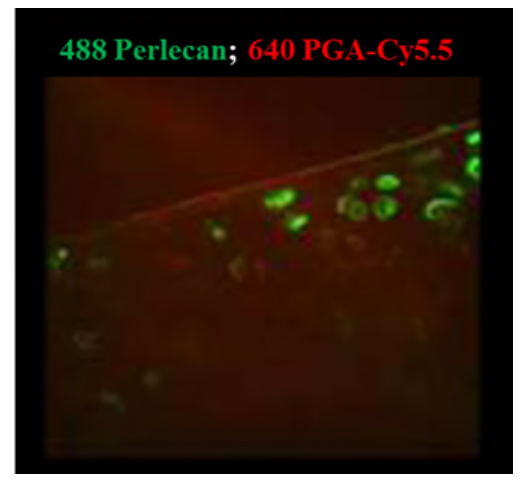

Figure 2. Pharmacokinetics of P-18 and PGA-P-18, and cartilage retention of PGA-P-18. (A) C57BL/6 mice were injected with the corresponding probe $(5 \mathrm{nM})$ i.v. in the tail vein. At various time points, blood samples (whole blood) were collected from the retro orbital sinus under anesthesia and the \%ID was determined. P-18 (diamonds); PGA-P-18 (squares). (B) Logarithmic plot of data reported in (A), revealing half-life of distribution $\left(\mathrm{t}_{1 / 2} \alpha\right)$ and clearance $\left(\mathrm{t}_{1 / 2} \beta\right)$. (C) Ex-vivo fluorescence (MFI, a.u) in mouse heart, spleen, kidney, liver, left knee and right knee (DMM-operated) of at 24 hours after i.v. injection of PGA-Cy5,5 in comparison with Cy5.5 alone (Control). (D) Histological section detected in mouse knee cartilage at 24 hours after injection of PGA-Cy5.5. Perlecan was detected in the cartilage using rat anti-perlecan and anti-rat Alexa Fluor 488. 
A

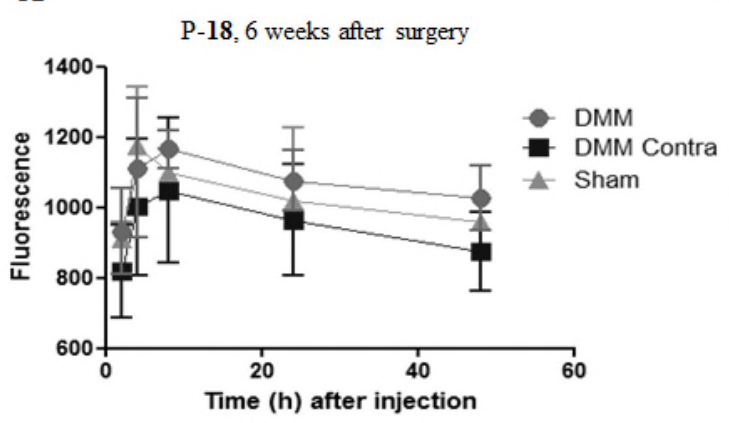

B

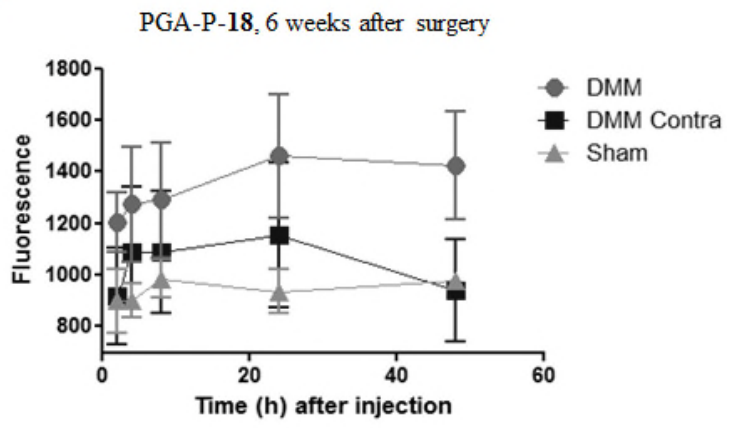

C

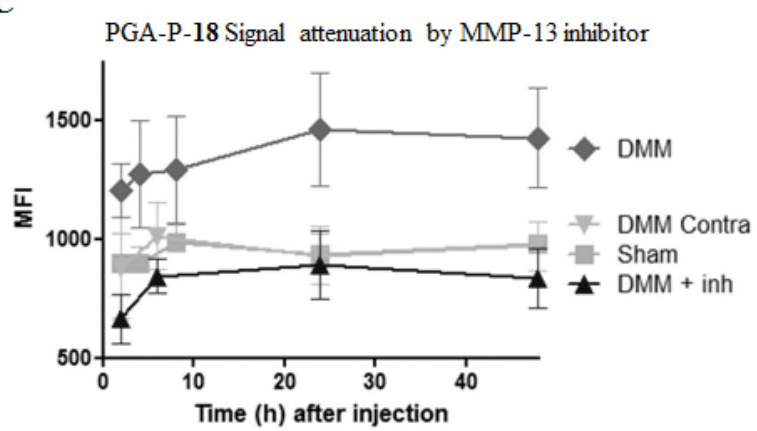

Figure 3. Fluorescence intensity due to probe activation in vivo after i.v. injection of the probes over time. A) P-18, 6 weeks after surgery, $n=3$. Paired t test: DMM vs. DMM contralateral, $\mathrm{p}<0.05$; DMM vs. Sham, $\mathrm{p}=0.29$ (n.s.). B) PGA-P-18 Probe, 6 weeks after surgery, $n=3$. Paired t test: DMM vs. DMM contralateral, $\mathrm{p}=0.0053$; DMM vs. Sham, $\mathrm{p}=0.0008$. C) Fluorescence intensity (MFI) registered for PGA-P-18 over time at 6 weeks post-surgery and influence of specific MMP-13 inhibitor A4727. Inhibitor was administered orally at a dose of $30 \mathrm{mg} / \mathrm{kg}$ daily before and 4 hours before imaging. $\mathrm{n}=5$ 
A

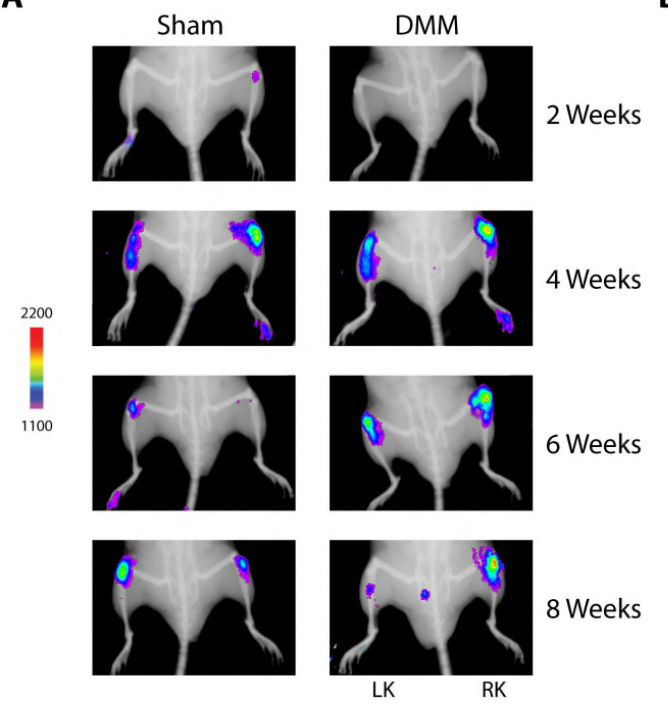

B

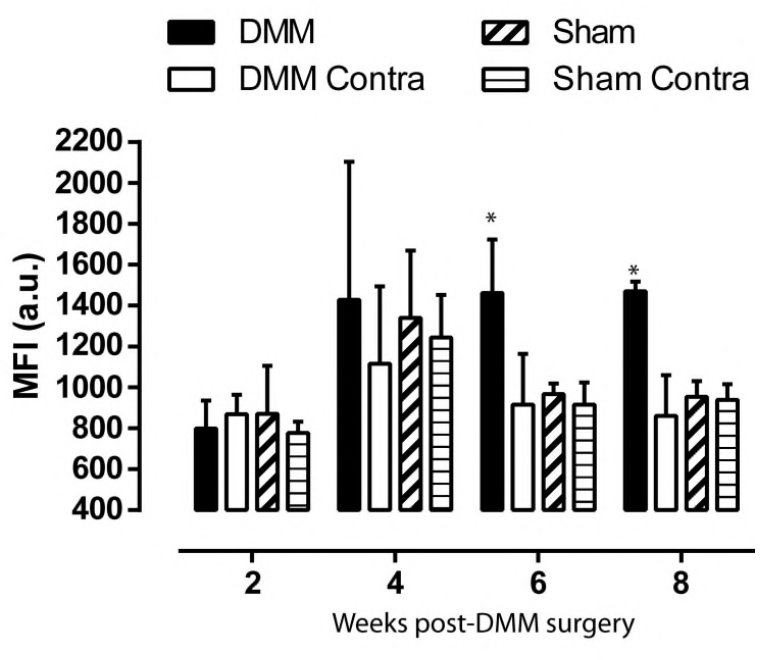

Figure 4. Activation of PGA-P-18 in vivo following DMM surgery. (A) Representative in vivo color images. Activation images of PGA-P-18 in mice following DMM or sham surgery were taken two days after i.v. injection of the probe at the time indicated postDMM surgically. Black and white X-ray images were used to localize the signal to the knee joints. (B) Quantification of the amount of PGA-P-18 activation as mean fluorescence intensity (MFI, a. u.) in the knee joints of DMM and sham-operated animals at the times indicated post-DMM surgery. Data are mean $\pm \mathrm{SD}, \mathrm{n}=5, * \mathrm{p}<0.05$ between DMM operated and other groups, two-way ANOVA with Bonferroni post tests. 
A

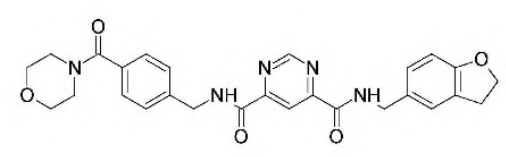

C

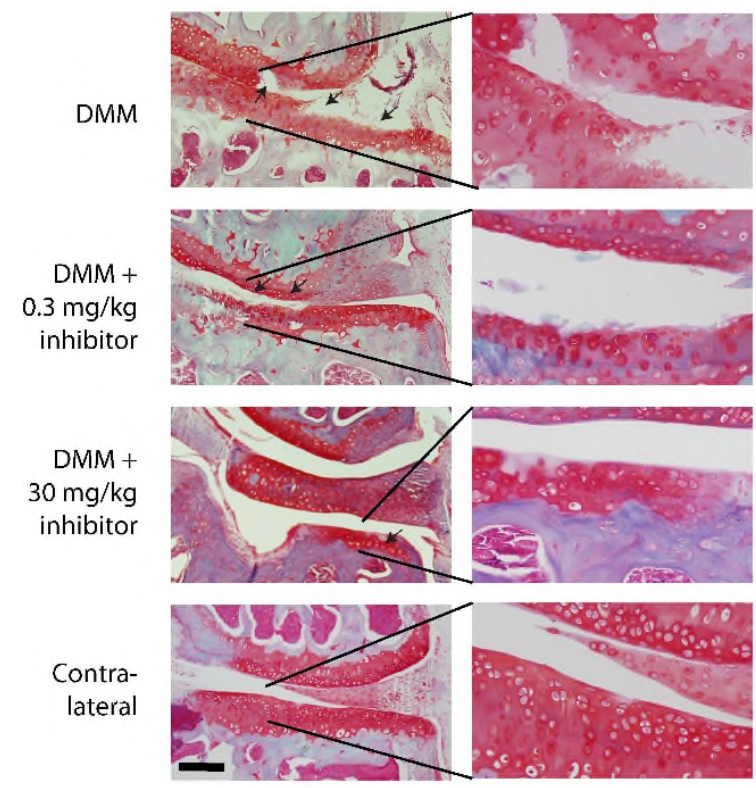

B

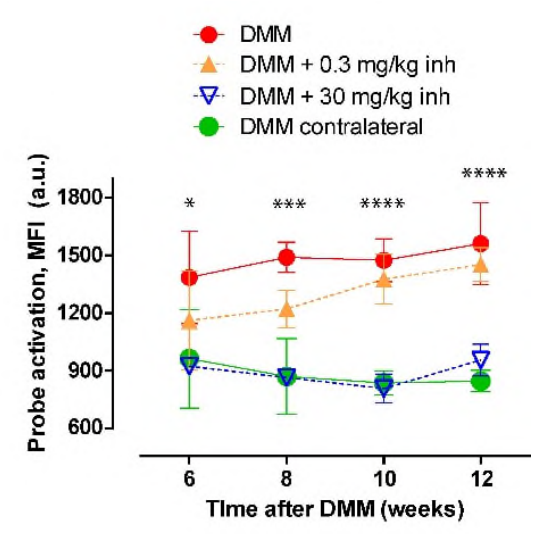

D

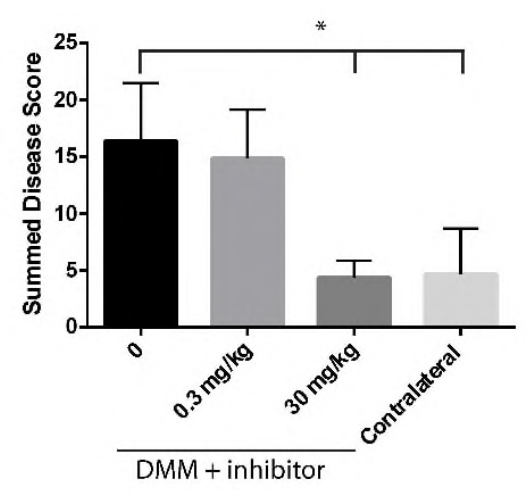

Figure 5. In vivo evaluation of the efficacy of MMP-13 inhibitor A4727 in the OA model. (A) Chemical structure of MMP-13 inhibitor A4727. (B) Activation of the PGA-P-18 probe after the DMM surgery in the knees of mice receiving no inhibitor (DMM and DMM contralateral $)$, a suboptimal dose $(0.3 \mathrm{mg} / \mathrm{kg})$ and optimal dose of MMP-13 inhibitor A4727 (30 mg/kg). (C) Representative histology of the damage observed in the mouse knee joints at 12 weeks after the DMM surgery. DMM, surgery only; DMM + inhibit, DMM surgery treated with daily dose of MMP-13 inhibitor A4727; Contralateral, contra-lateral knee of the mice treated with $30 \mathrm{mg} / \mathrm{kg}$ inhibitor. Black arrows indicate areas of aggrecan degradation. (D) Summed disease scores as determined by histology at 12 weeks after DMM surgery. Data are mean $\pm \mathrm{SD}, \mathrm{n}=5 . * \mathrm{p}<0.05$, *** $\mathrm{p}$ $<0.005$, **** $\mathrm{p}<0.001$ between DMM and $30 \mathrm{mg} / \mathrm{kg}$ groups after two-way ANOVA with Bonferroni post tests. 
Table 1. Inhibition profile of A4727 on MMPs, apparent $K_{i \text { app }}$ values.

\begin{tabular}{|c|c|c|c|c|c|c|}
\hline $\begin{array}{l}\text { MMP- } \\
1\end{array}$ & $\begin{array}{l}\text { MMP- } \\
2\end{array}$ & $\begin{array}{l}\text { MMP- } \\
3\end{array}$ & $\begin{array}{l}\text { MMP- } \\
8\end{array}$ & $\begin{array}{l}\text { MMP- } \\
9\end{array}$ & $\begin{array}{l}\text { MMP- } \\
13\end{array}$ & $\begin{array}{l}\text { MMP- } \\
14\end{array}$ \\
\hline$>6,500$ & $>6,500$ & $>6,500$ & $>6,500$ & $>6,500$ & $8 \mathrm{nM}$ & $>6,500$ \\
\hline $\mathrm{nM}$ & $\mathrm{nM}$ & $\mathrm{nM}$ & $\mathrm{nM}$ & $\mathrm{nM}$ & & $\mathrm{nM}$ \\
\hline
\end{tabular}


An in vivo optical imaging probe comprised of a FRET peptide specific for MMP13 conjugated to poly-L-glutamic acid allows live In vivo Imaging of Disease-Related Events. The nanometric probe detects early stages of the disease in a murine surgical model of osteoarthritis and provides real time readout on therapeutic efficacy of MMP13 inhibitors.

Keyword: MMP-13 specific nanometric probe

Aroa Duro-Castano ${ }^{\dagger}$, Ngee Han $\operatorname{Lim}^{\dagger}$, Isabelle Tranchant ${ }^{\dagger}$, Mehdi Amoura, Fabrice Beau, Heike Wieland, Otmar Kingler, Matthias Herrmann, Marc Narazé, Oliver Plettenburg, Vincent Dive*, María J. Vicent", Hideaki Nagase

${ }^{\dagger}$ These authors contributed equally to this work

In Vivo Imaging of MMP-13 Activity Using A Specific Polymer-FRET Peptide Conjugate Detects Early Osteoarthritis And Inhibitor Efficacy

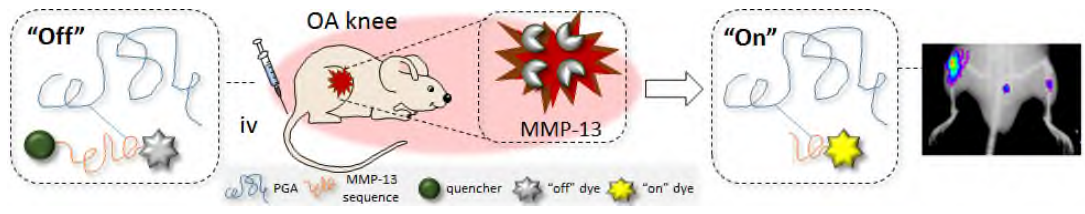




\section{Supporting Information}

In Vivo Imaging of MMP-13 Activity Using A Specific Polymer-FRET Peptide Conjugate Detects Early Osteoarthritis And Inhibitor Efficacy

Aroa Duro-Castano ${ }^{\dagger}$, Ngee Han Lim ${ }^{\dagger}$, Isabelle Tranchant ${ }^{\dagger}$, Mehdi Amoura, Fabrice Beau, Heike Wieland, Otmar Kingler, Matthias Herrmann, Marc Narazé, Oliver Plettenburg, Vincent Dive*, María J. Vicent ${ }^{*}$,Hideaki Nagase*

${ }^{\dagger}$ These authors contributed equally to this work 


\section{INDEX}

\section{Supporting materials and methods}

1.1. Materials

1.2. Characterization techniques

1.2.1. Probe characterization

RP-HPLC

MALDI-TOF/TOF

Ultraviolet (UV)-Visible Spectroscopy

1.2.2. Polymer characterization

Gel Permeation Chromatography (GPC)

Nuclear Magnetic Resonance (NMR)

Circular Dichroism (CD)

Z-Potential measurements

UV-Visible Spectroscopy

\subsection{Synthetic protocols}

1.3.1. Probes synthesis

Compounds 1, 2, 19 and 20

Compounds 3, 7, 8, 9, 10, 11 and 12:

Compounds 4, 5 and 6

Compounds 13, 14, 15, 16 and 17

Compound 18

1.3.2. Polymer synthesis

1.3.3. Synthesis of MMP-13 inhibitor A4727

1.3.4. Pharmacokinetics of P-18 and PGA-P-18

1.3.5. Enzyme kinetics Analysis

1.3.6. Animal model studies

1.3.7. Distribution of PGA after i.v. injection

1.3.8. Activation of probes in vivo

1.3.9. Real time readout of the effect of MMP-13 inhibitor in the DMM surgery model of OA

\section{Supporting Figures}

1.3.10. Histology and scoring of cartilage damage

Figure S1. A) chemical structure of the probes, modifications have been introduced in the P1' position of the probe using the different reported side chains ( $\mathrm{R}$ groups); B) catalytic efficiency $\left(k_{c a t} / K_{M}\right)$ of recombinant MMP-13 in cleaving probes at $\mathrm{pH} 6.8$ and $25^{\circ} \mathrm{C}$ (25 mM HEPES, $1 \mathrm{mM} \mathrm{CaCl} 2,0.2 \mathrm{M} \mathrm{NaCl}$; probe concentration $0.5 \mathrm{mM}$ :MMP$13,1 \mathrm{nM})$.

Figure S2. Catalytic efficiency $\left(\mathrm{k}_{\mathrm{ca}} / \mathrm{K}_{\mathrm{M}}\right)$ of recombinant MMPs and cathepsins in cleaving the probe $\mathrm{P}-18$ at $\mathrm{pH} 6.8$ and $25^{\circ} \mathrm{C}\left(25 \mathrm{mM}\right.$ Hepes, $1 \mathrm{mM} \mathrm{CaCl}_{2}, 0.2 \mathrm{M} \mathrm{NaCl}$; probe concentration $0.5 \mathrm{mM}$; enzymes, $1 \mathrm{nM}$

Figure S3. Distribution of PGA-Cy5.5 compared with Cy5.5 (Control) alone. Mice were i.v. injected with either Cy5.5 alone or PGA-Cy5.5, and at 24 hours post injection in comparison ex-vivo fluorescence (MFI, a.u) was monitored in Heart, Spleen, Kidney, Liver, Left Knee and Right Knee (DMM).

Figure S4. A) progress curves of degradation of the PGA-18 probe by MMPs; in diluted mouse plasma (1/10 dilution), in the buffer $\left(\mathrm{pH} 6.8,25^{\circ} \mathrm{C}, 25 \mathrm{mM}\right.$ Hepes, $1 \mathrm{mM} \mathrm{CaCl}_{2}$, $0.2 \mathrm{M} \mathrm{NaCl}$; probe concentration $0.5 \mathrm{mM}$; enzymes, $1 \mathrm{nM})$. In control experiment $(\mathrm{Ctl})$, 
no MMP was added, showing the probe stability under these conditions. B) $k_{c a} / K_{M}$ values obtained from the enzymatic curves in diluted mouse plasma.

Figure S5. Activation of P-18 and PGA-P-18 in vivo at 8 weeks following DMM surgery. (A) Activation of P-18 following i.v. injection; (B) Activation of PGA-P-18 following i.v. injection; (C) Inhibition of activation of PGA-P-18 in the presence of the MMP-13 inhibitor. Data are mean $\pm \mathrm{SD}, \mathrm{n}=5$. * $\mathrm{p}<0.05, * \mathrm{p}<0.01$, *** $\mathrm{p}<0.005$, **** $\mathrm{p}<$ 0.001 between DMM and other groups (A, B), and between P-18 and PGA-P-18 (C) after two-way ANOVA with Bonferroni post tests.

\section{Supporting Tables}

Table S1. HPLC and mass-spectrometry data.

Table S2. Characterization of PGA precursors and conjugate.

\section{Supporting Materials and Methods}

\subsection{Materials}

Commercial reagents were used as received without additional purification. Solvents were of the reagent grade available. Fmoc amino acids, rink amide resin, universal PEG NovaTagTM resin and 1-[(1-(cyano-2-ethoxy-2-oxoethylideneaminooxy) dimethylaminomorpholino)] uronium hexafluorophosphate (COMU) were from Novabiochem. N- $\alpha$-Fmoc-N- $\beta$-(2,4-dinitrophenyl)-L-2,3-diaminopropionic acid (FmocDap(Dnp)-OH), Fmoc-4- fluoro-L-phenylalanine and (7-methoxycoumarin- 4-yl)acetyl (Mca) were from Bachem. Fmoc-4-nitrophenylalanine, Fmoc-4-bromo-L-phenylalanine, Fmoc-4-chloro-L-phenylalanine and Fmoc-4-(phenoxy)-L-phenylalanine were from PolyPeptide. Fmoc-4-iodo-L-phenylalanine, Fmoc-propargyl glycine, 1hydroxybenzotriazole (HOBt), dichloromethane (DCM), N,N-dimethylformamide (DMF), anhydrous tetrahydrofuran (THF), phenylboronic acid, tetrakis(triphenylphosphine)palladium, 4-bromobiphenyle, sodium ascorbate, sodium azide, N,N-dimethylethylenediamine, copper iodide, copper sulphate, neopentyl amine, 2-amino-2-(hydroxymethyl)-1,3-propanediol (Tris base) and calcium chloride $\left(\mathrm{CaCl}_{2}\right)$ 
were from Sigma-Aldrich. N,N-diisoproylethylamine was from Fluka. $\mathrm{NH}_{2}-\mathrm{EG}(6)-\mathrm{N}_{3}$ and H-L-Glu(OBzl)-OH were obtained from Iris Biotech. Deuterated chloroform- $d 1$, DMSO- $d 6$, DMF- $d 7$ and $\mathrm{D}_{2} \mathrm{O}$ were purchased from Deutero $\mathrm{GmbH}$. Preparative SEC was performed using Sephadex G-25 superfine from GE as well as PD MiniTrap G-10 TM columns containing $2.1 \mathrm{~mL}$ of Sephadex ${ }^{\mathrm{TM}} \mathrm{G}-10$. Dialysis was performed in a Millipore ultrafiltration device fitted with a 1, 3, 5, $10 \mathrm{kDa}$ MWCO (Vivaspin®) regenerated cellulose membrane.

C57BL/6J mice (10 week old male) were obtained from Harlan Laboratories, Blackthorn, Bicester, UK.

\subsection{Characterization techniques}

\subsubsection{Probe characterization}

RP-HPLC: Analytical and preparative RP-HPLC separations were performed on a Shimadzu HPLC system using analytical AIT C8 Kromasil $(250 \times 4,6$ mm, $100 \AA$, gradient 0 to $20 \mathrm{~min} / 0$ to $100 \% \mathrm{~B}$, flow rate $1 \mathrm{~mL} \cdot \mathrm{min}-1$ ) and semi-preparative AIT C8 Kromasil $(250 \times 20 \mathrm{~mm} 100 \AA$, gradient 0 to $30 \mathrm{~min} / 0$ to $100 \% \mathrm{~B}$, flow rate $3 \mathrm{~mL}$.min1) columns, UV detection was performed at $215 \mathrm{~nm}$ et $330 \mathrm{~nm}$. Solvent systems used, A: 0,1 \% TFA in water and B: 0,09\% TFA in acetonitrile.

MALDI-TOF/TOF: A 4800 spectrometer MALDI-TOF/TOF Proteomics Analyzer (Applied Biosystems, Foster City, CA) was used for mass spectrometry analysis of samples. $\alpha$-Cyano-4-hydroxycinnamicacid (HCCA) from Fluka was used as organic matrix for mass spectrometry analysis. Cyanine substrate was analyzed on a hybrid instrument LTQ-ORBITRAPTM_ThermoElectron Corporation. 
Ultra-Violet (UV)-Visible Spectroscopy: UV measurements were performed with a Shimadzu spectrophotometer (UV 1800).

\subsubsection{Polymer Characterization}

Gel Permeation Chromatography (GPC): For SEC measurements in DMF containing 1g. L-1 of lithium bromide as an additive, an Agilent 1100 series system was used with a flow rate of $1 \mathrm{~mL} \cdot \mathrm{min}^{-1}$ at $30^{\circ} \mathrm{C}$ as an integrated instrument, including three HEMAbased columns (105/103/102 A porosity) from MZ-Analysentechnik GmbH, a UV (275 nm) and an RI detector. Calibration was achieved with well-defined Poly(methyl methacrylate) (PMMA)/DMF standards, provided by Polymer Standards Service (PSS)/Mainz Germany.

Nuclear Magnetic Resonance (NMR): ${ }^{1} \mathrm{H}-\mathrm{NMR}$ spectra were obtained at 300 or $400 \mathrm{MHz}$ using a FT-spectrometer from Bruker and analyzed using the MestreNova 6.2 software. Diffusion Experiments: Pulsed field gradient NMR spectroscopy was used to measure translational diffusion by fitting the integrals or intensities of the NMR signals to the Stejskal-Tanner ${ }^{[44]}$ using the equation: $\mathrm{I}=\mathrm{I} 0 \exp [-\mathrm{D} \gamma 2 \mathrm{~g} 2 \delta 2(\Delta-\delta / 3)]$, where $\mathrm{I}$ is the observed intensity, I0 the reference intensity (unattenuated signal intensity), D the diffusion coefficient, $\gamma$ the gyromagnetic ratio of the observed nucleus, $g$ the gradient strength, $\delta$ the length of the gradient, and $\Delta$ the diffusion time. Two-dimensional diffusion-ordered NMR spectroscopy (DOSY) was performed with a stimulated echo sequence using bipolar gradient pulses. The lengths of delays were held constant at $\Delta=$ $100 \mathrm{~ms}$, and 32 spectra of 64 scans each were acquired with the strength of the diffusion gradient varying between $5 \%$ and $95 \%$. The lengths of the diffusion gradient and the stimulated echo were optimized for each sample. Typical values were $\delta=1.5 \mathrm{~ms}$.

Circular Dichroism (CD): CD Spectroscopy was performed with a J-815 CD Spectrometer (JASCO Corporation) using a Peltier thermostated cell holder (PTC-423, 
JASCO Corporation) with a recirculating cooler (JULABO F250, JASCO Corporation). A nitrogen flow ( 2.7 L.min-1) was lead through the spectrometer and controlled with a nitrogen flow monitor (Afriso Euro-Index). The samples were dissolved in HFIP for protected and water for deprotected samples and diluted to a concentration of $0.1 \mathrm{mg} \cdot \mathrm{mL}^{-}$ ${ }^{1}$. Samples were measured repeatedly $(n=3)$ in a quartz cuvette with $\mathrm{d}=0.1 \mathrm{~cm}$ at $20{ }^{\circ} \mathrm{C}$. Obtained molar ellipticities were plotted as mean residue ellipticity.

Z-Potential measurements: Z-potential measurements were performed at $20{ }^{\circ} \mathrm{C}$ using a Malvern ZetasizerNanoZS instrument, equipped with a $532 \mathrm{~nm}$ laser using Disposable folded capillary cells, provided by Malvern Instruments Ltd. (Worcerstershire, UK). Polymer solutions were prepared in $\mathrm{ddH}_{2} \mathrm{O}$. The solutions were filtered through a 0.45 $\mu \mathrm{m}$ cellulose membrane filter. Z-potential was measured for each sample per triplicate with $\mathrm{n}>3$ measurements.

UV-VIS spectroscopy: UV-VIS measurements were performed using JASCO V-630 spectrophotometer at $25^{\circ} \mathrm{C}$ with $1.0 \mathrm{~cm}$ matched quartz cells and with spectral bandwidth of $0.5 \mathrm{~nm}$.

\subsection{Synthetic protocols}

\subsubsection{Probes synthesis}

Compounds 1, 2, 19 and 20 


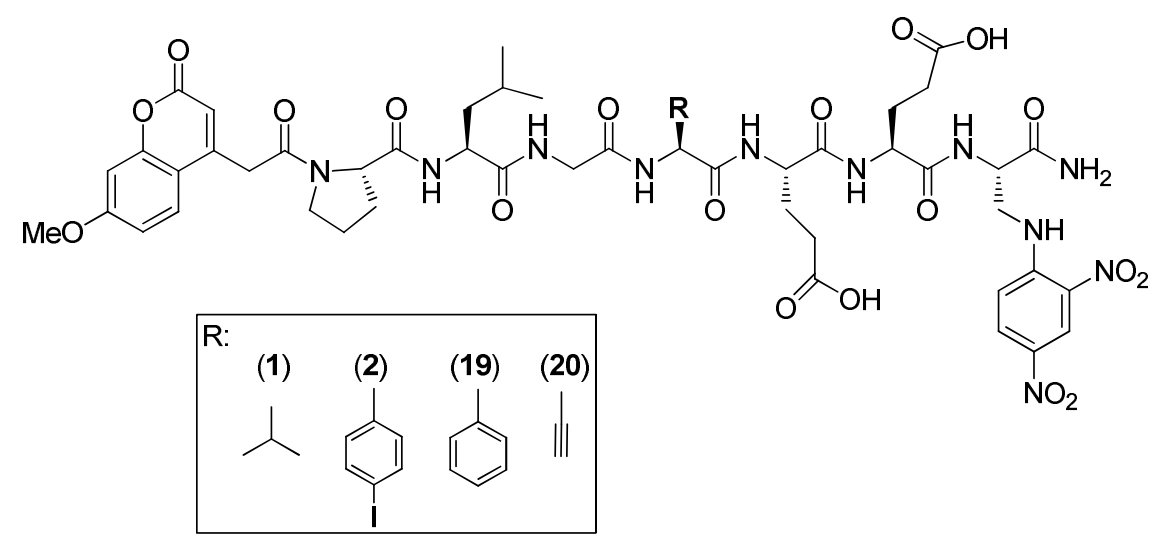

Scheme S1. MMP-13 selective probes 1, 2, 19 and 20.

Probes with the general structure reported in Scheme S1 were synthesized by Fmoc solidphase synthesis strategy. Rink amide resin was swelled with dichloromethane (DCM) (15 min). Removal of Fmoc from Rink amide resin was performed using piperidine in N,N'Dimethylformamide (DMF) (20\% piperidine, 2 x $2 \mathrm{~min}, 1 \times 5 \mathrm{~min})$, then $\mathrm{N}-\alpha-\mathrm{Fmoc}-\mathrm{N}-\beta$ (2,4-dinitrophenyl)-L-2,3-diaminopropionic acid (Fmoc-Dap(Dnp)-OH), was incorporated into the solid support using COMU® ((1-Cyano-2-ethoxy-2oxoethylidenaminooxy)dimethylamino-morpholino-carbenium hexafluorophosphate) as coupling agent for 30 minutes (6 equivalents of amino-acid, 5.8 equivalents of COMUß, 12 equivalents of $\mathrm{N}, \mathrm{N}-$ Diisopropilethylamina, DIPEA, and DMF). A second coupling was performed for 20 minutes ( 3 equivalents of amino-acid, 2.8 equivalents of COMUß, 6 equivalents of DIPEA, DMF). Then, after sequential incorporation of each amino acid of the peptide sequence, the fluorophore (7-methoxycoumarin-4-yl)acetyl was coupled (6 equivalents of amino-acid, 5.8 equivalents of COMU®, 12 equivalents of DIPEA, DMF) for 1 hour and a second coupling was performed. After cleavage of the resin (Trifluoroacetic Acid (TFA)/ Triisopropylsilane (TIS)/ $\mathrm{H}_{2} \mathrm{O}, 95 / 2.5 / 2.5,4$ hours) and freeze-drying of the cleavage solution, crude product was purified by HPLC (0-100 \% B in 30 minutes). After freeze-drying of the purified fractions and addition of Dimethyl sulfoxide (DMSO), the probe concentration was determined by UV $(\lambda=333 \mathrm{~nm} ; \varepsilon=21$ 
$470 \mathrm{~cm}^{-1} \mathrm{M}^{-1}$ ). Product purity was assessed by analytical HPLC and mass spectrometry analysis, reported in Table S1.

Compounds 3, 7, 8, 9, 10, 11 and 12:

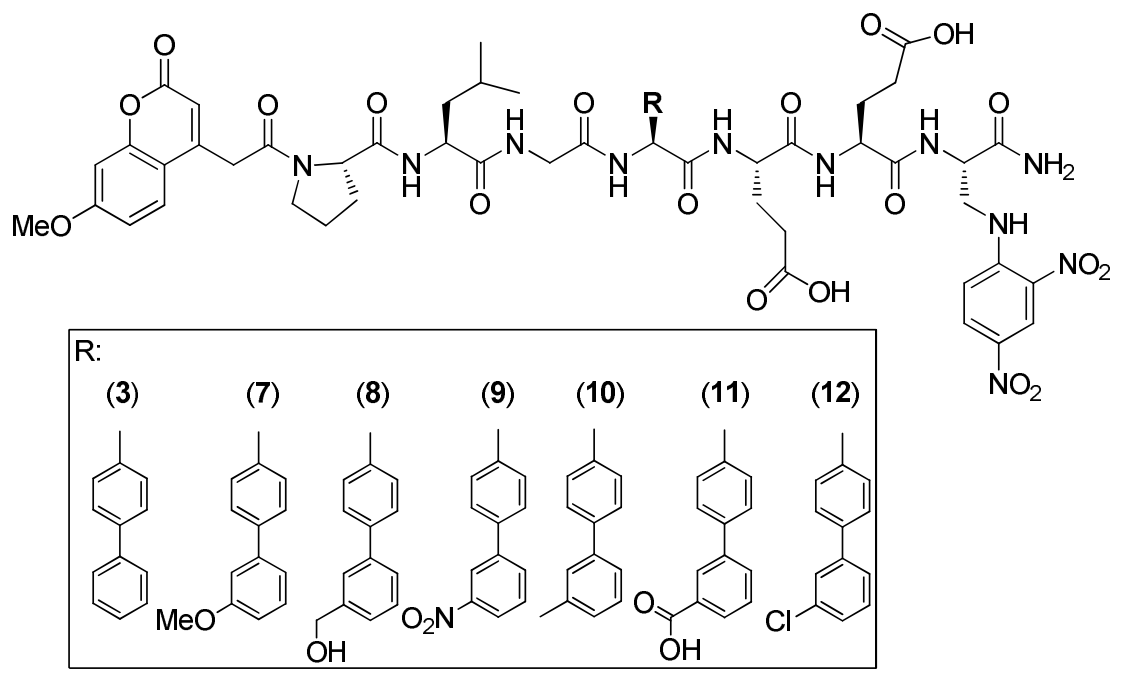

Scheme S2. MMP-13 selective probes 3, 7, 8, 9, 10, 11 and 12.

Probes with the general structure from Scheme S2 were prepared as follows. To prepare these compounds, a Suzuki coupling was performed starting from compound 19 (R=Phe) still on the resin, as prepared above, on which was added the corresponding boronic acid (10 eq, $0.2 \mathrm{M}$ in $\mathrm{DMF})$, potassium carbonate (10 eq, $0.16 \mathrm{M}$ in water) and Tetrakis (triphenylphosphine) palladium (1 eq., $0.08 \mathrm{M}$ in DMF). The reaction mixture was irradiated in a microwave apparatus at $80{ }^{\circ} \mathrm{C}, 300 \mathrm{~W}$ for $10 \mathrm{~min}$. The resin was then washed with DMF and DCM and cleavage from the resin was performed as described in general procedure. Product purity was assessed by analytical HPLC and mass spectrometry analysis, reported in Table S1.

Compounds 4, 5 and 6: 


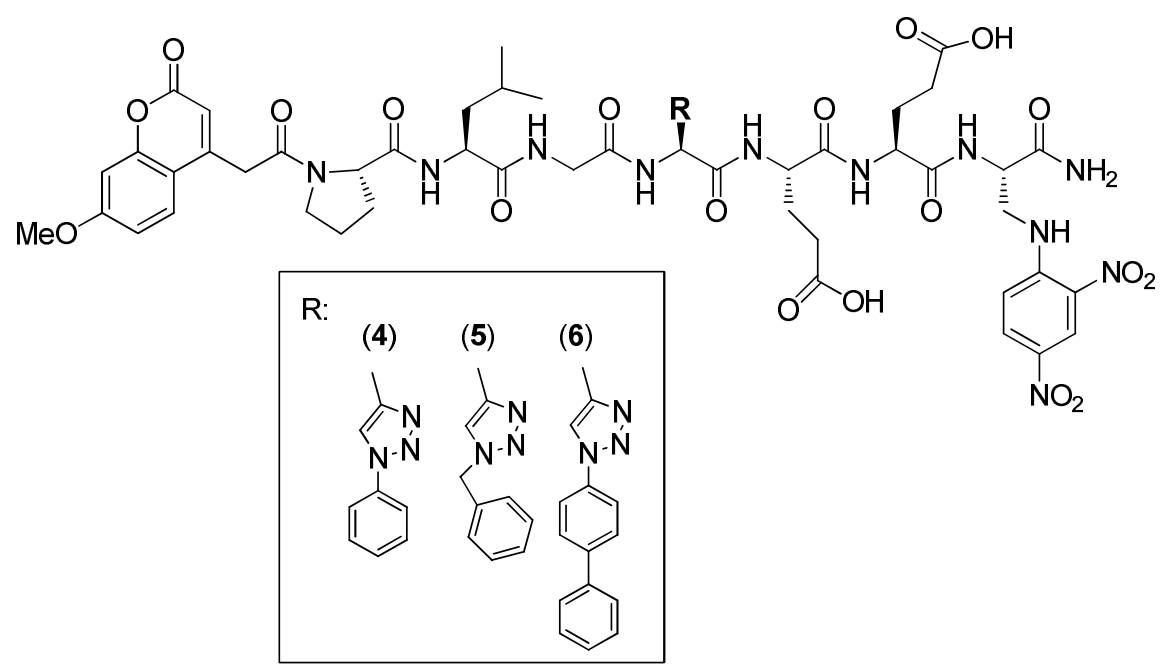

Scheme S3. MMP-13 selective probes 4, 5 and 6.

Probes with the general structure depicted in Scheme S3 were prepared as follows. Starting from compound 20 still on the resin, a cyclo-addition addition was preformed using the following bromo-derivatives (10 eq, 4-bromophenyl, compound 4; 4bromobenzyl, compound 5 and 4-bromobiphenyle, compound 6), then adding sodium ascorbate (20 eq., $0.9 \mathrm{M}$ in water), sodium azide (20 eq., $0.9 \mathrm{M}$ in water), N,Ndimethylethylenediamine (10 eq.), copper iodide (10 eq., $0.18 \mathrm{~m}$ in anhydrous THF) were added on the resin. The reaction mixture was irradiated in a microwave apparatus at 90 ${ }^{\circ} \mathrm{C}, 150 \mathrm{~W}$ for $10 \mathrm{~min}$. The resin was then washed with DMF and DCM and cleavage from the resin was performed as described in general procedure. Product purity was assessed by analytical HPLC and mass spectrometry analysis, reported in Table S1.

Compounds $13,14,15,16$ and $17:$ 


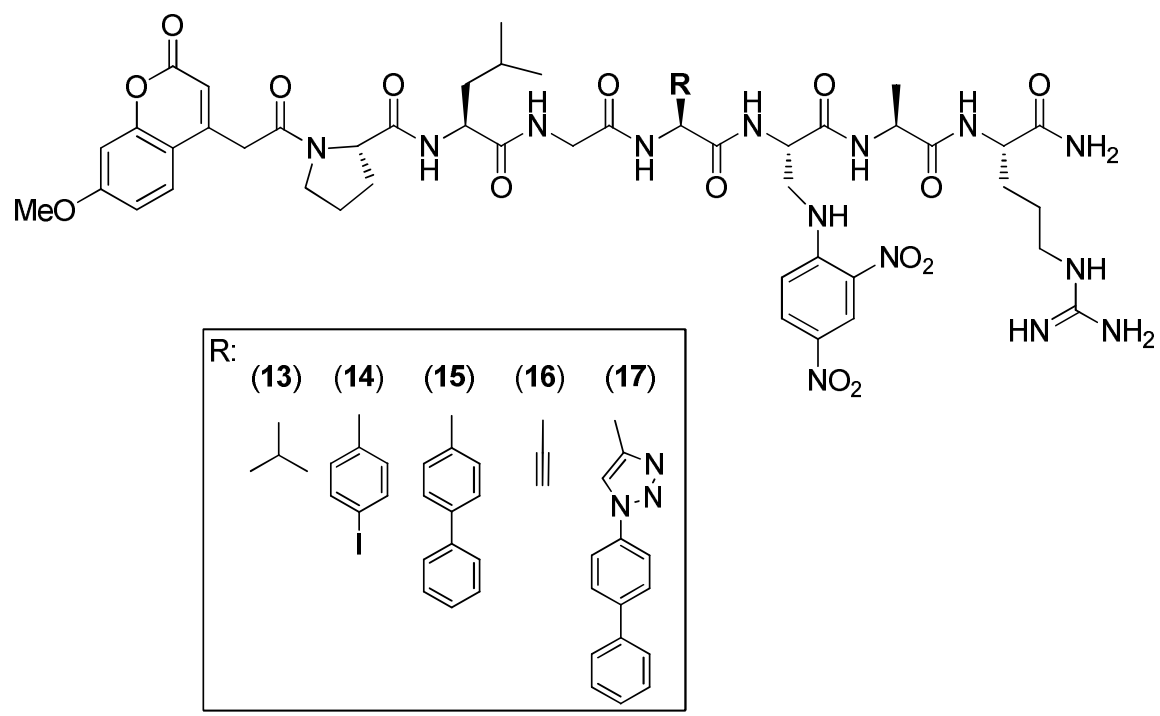

Scheme S4. MMP-13 selective probes 13, 14, 15, 16 and 17.

The compounds were prepared as described before, but starting from the general structure of Scheme S4. Specifically, compound 13, 14 and 16 were synthesized as for compounds 1,2 , and 20, respectively. Compound 15 was synthesized from compound 14 . Following the same procedure as for compound 3. Compound 17 was synthesized from compound 16 , following the same procedure as for compound 6 . 


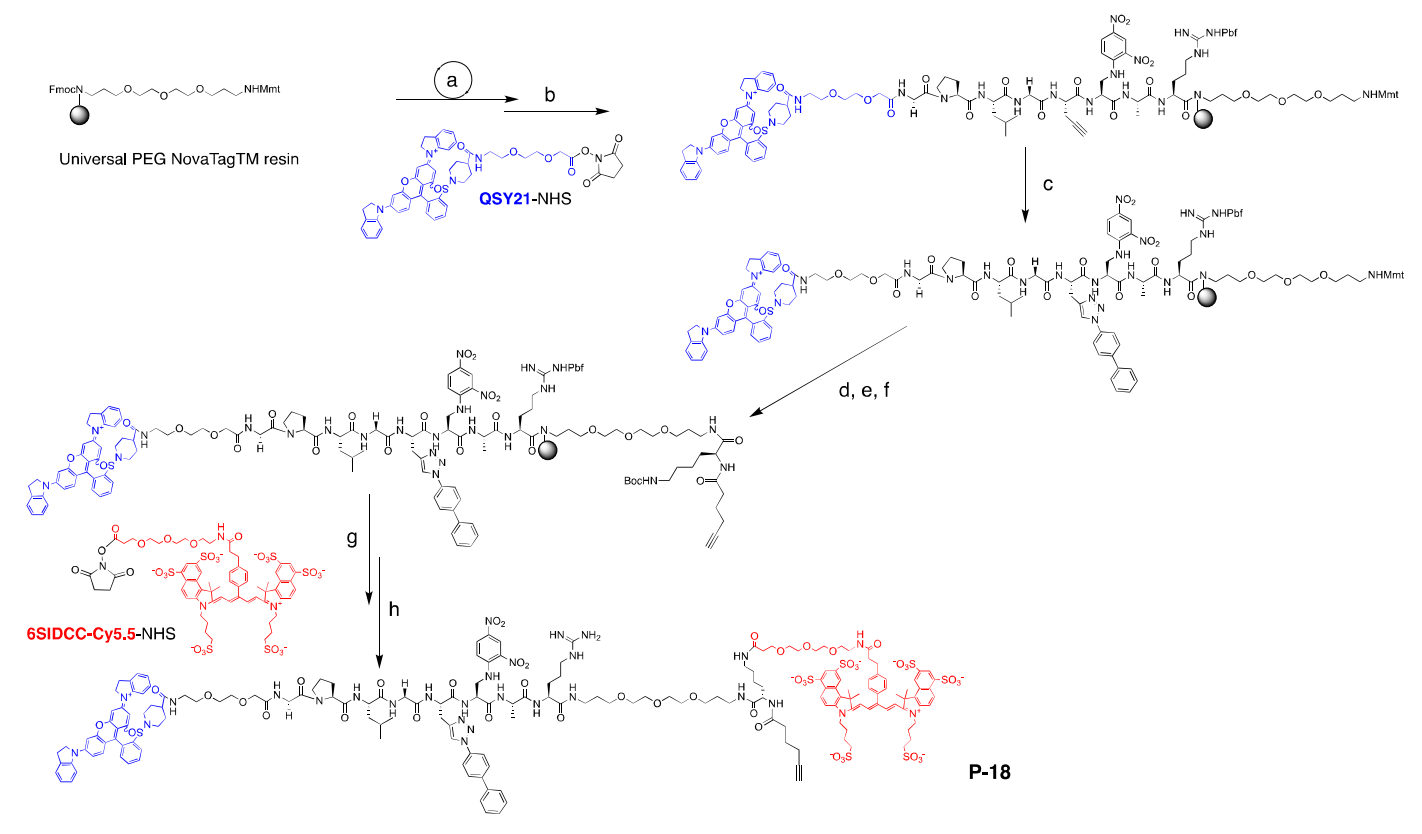

Reagents and conditions: (a) Fmoc solid phase synthesis, Fmoc-AA-OH (6 eq), COMU (5.8 eq), DIPEA (12 eq), DMF, $60^{\circ} \mathrm{C}, 45 \mathrm{~W}, 2 \times 2 \mathrm{~min}$, (b) QSY21-NHS (0.8 eq), DIPEA (16 eq), HOBt (0.5 eq), DMF, RT, 24h, (c) 4-Bromobiphenyle (20 eq), Sodium ascorbate (20 eq, $0.9 \mathrm{M}$ aq), sodium azide (20 eq, $0.9 \mathrm{M}$ aq), N,N dimethylenediamine (20 eq)

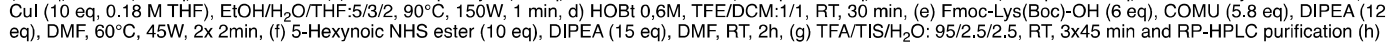
6 SIDCC-Cy5.5-NHS (0.8 eq), DIPEA (16 eq), HOBt (0.5 eq), DMF, RT, $24 \mathrm{~h}$

Scheme S5. Synthetic pathway for P-18.

Standard Fmoc methodology was used to build the amino acid sequence on a Universal PEG NovaTagTM resin (Novabiochem). Fmoc removal and amino acids incorporation were performed as described (see synthesis of compounds 1 to 17). After Fmoc removal under microwave irradiation (piperidine $20 \%$ in DMF, $2 \times 2 \mathrm{~min}, 60^{\circ} \mathrm{C}, 25 \mathrm{~W}$ ), NHSactivated quencher QSY21 (0.8 eq., Invitrogen) was conjugated to the resulting N-ter amino free moiety in presence of $N, N$-Diisopropylethylamine (DIPEA, 16 eq) and 1Hydroxybenzotriazole (HOBt, 0.5 eq.) in $\mathrm{DMF}$ at room temperature for $24 \mathrm{~h}$. After washing $(3 \times$ DMF followed by $3 \times \mathrm{DCM})$, a 1,3-dipolar cycloaddition was performed following the protocol described for compound $\mathbf{6}$. The monomethoxytrityl protecting group (Mmt) was subsequently removed at room temperature using a solution of $0.6 \mathrm{M}$ HOBt (Trifluoroethanol/Dichloromethane 1:1). After $30 \mathrm{~min}$ and a washing cycle $(2 \times$ 
DMF and $2 \times$ DCM), Fmoc-Lys(Boc)-OH (6 eq) preliminarily activated with COMU (5.8 eq) and DIPEA (12 eq) in DMF, was added and the reaction was carried out at room temperature for $1 \mathrm{~h}$. After Fmoc removal (piperidine $20 \%$ in DMF, $2 \times 2 \mathrm{~min}, 60^{\circ} \mathrm{C}, 25 \mathrm{~W}$ ), 5-hexynoic NHS ester (10 eq.) first synthesized in solution was conjugated in presence of DIPEA (15 eq.) at room temperature. After an overnight gentle stirring, the resulting peptide was cleaved from the support with Tri-fluoroacetic acid (TFA)/Triisopropylsilane (TIS)/water 95:2.5:2.5 cocktail $(3 \times 45 \mathrm{~min})$ and the crude was purified by RP-HPLC on a Supelco Ascentis ${ }^{\circledR}$ C18 column $(150 \times 10 \mathrm{~mm}, 5 \mu \mathrm{m}$, flow rate $=3 \mathrm{~mL} / \mathrm{min})$ using the following program: 0 to $100 \% \mathrm{~B}$ in 30 min with $\mathrm{A}: \mathrm{H}_{2} \mathrm{O} / 0.1 \%$ TFA and $\mathrm{B}$ : Acetonitrile/0.09\% TFA as eluents. UV detection was performed at $230 \mathrm{~nm}$ and $280 \mathrm{~nm}$. After freeze-drying, the resulting intermediate was engaged in a coupling reaction with NHS-activated 6SIDCC-Cy5.5 (0.8 eq., Mivenion) in presence of (DIPEA, 16 eq.) and HOBt $(0,5$ eq) in DMF at room temperature. The reaction was monitored by analytical HPLC using a Kromasil C8 column $(150 \times 4.6 \mathrm{~mm}, 5 \mu \mathrm{m}$, flow rate $=1 \mathrm{~mL} / \mathrm{min})$ with the following program: 0 to $100 \% \mathrm{~B}$ in 20 min with $\mathrm{A}: \mathrm{H}_{2} \mathrm{O} / 0.1 \%$ TFA and $\mathrm{B}$ : Acetonitrile/0.09\% TFA as eluents. UV detection was performed at $230 \mathrm{~nm}$ and $280 \mathrm{~nm}$. After reaction completion, P18 was purified on silica gel C8-reversed phase (60759, Aldrich) using the following protocol. For 10-15 mg of crude P18 use 50g of silica gel. After suspension in $100 \%$ acetonitrile and transfer into an appropriate column, silica gel was first conditioned with acetonitrile $/ \mathrm{H}_{2} \mathrm{O}: 1 / 1$ (3 equivalent volumes relative to the volume of silica gel) and then with $100 \% \mathrm{H}_{2} \mathrm{O}$. P18 was loaded and eluted with a mobile phase whose acetonitrile content increased gradually. P18 was eluted from the column at $50 \%$ of acetonitrile and resulted after freeze-drying in a blue solid (7\% yield) that was stored at $-20^{\circ} \mathrm{C}$ away from light. 


\subsubsection{Polymer synthesis}

$\underline{\text { Polymer carrier synthesis. }}$

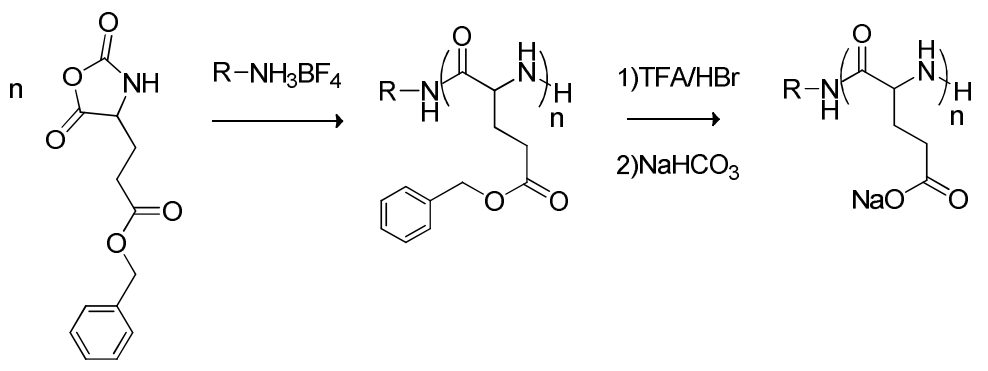

Scheme S6. Polyglutamic acid (PGA) sodium salt synthesis

Polyglutamic acid (PGA) $(\mathrm{Mn}=19600$, polydispersity $(Đ)=1.2,152$ Glutamic Acid Units $(\mathrm{GAU})$ ) was synthesized according to the previously published method (Scheme S6). ${ }^{[45]}$ Y-Benzyl-L-glutamate N-carboxyanhydride was polymerized by using Schlenk techniques, under $\mathrm{N}_{2}$ flow, with previously purified solvents, and recrystallized initiators (neopentyl ammonium $\mathrm{BF}_{4}$ salt). The polymerization reactions were left to proceed for 3 days at $40{ }^{\circ} \mathrm{C}$ and the products were checked for well-defined and narrowly distributed benzyl protected polyglutamate (PBLG) size after precipitation in diethyl ether and purification of the polymers. Monomodal distributions were achieved with low polydispersity (PDI < 1.1-1.2) and controlled molecular weights. Then, PGA was obtained by acidic deprotection. Briefly, PBLG was dissolved in TFA. Then 2 eq. of $\mathrm{HBr}$ $\left(48 \% \mathrm{v} / \mathrm{v}, 1.49 \mathrm{~g} \cdot \mathrm{cm}^{-3}, 81 \mathrm{~g} \cdot \mathrm{mol}^{-1}\right)$ per carboxylic group were added drop wise, and the yellow mixture was left stirring for 16 hours. The solution was then poured into a large excess of cold diethyl ether leading to a white solid that was recovered after centrifugation (2600 rpm, $4{ }^{\circ} \mathrm{C}, 10$ minutes). The product was washed per triplicate with diethyl ether and dried over high vacuum. After that, the product was then purified by acid-base precipitation $\left(\mathrm{NaHCO}_{3} / \mathrm{HCl} 6 \mathrm{M}\right)$. Dialysis or ultrafiltration was done leading to the sodium salt form. 
Polyglutamic acid modification with azides.

PGAcoEG(6) $\mathrm{N}_{3}$ was synthesized by post polymerization modification according to the previously published method ${ }^{[27]}$ (Scheme S7). Briefly, the introduction of azide functionalities was developed by using 4-(4,6-Dimethoxy-1,3,5-triazin-2-yl)-4-methyl morpholinium chloride (DMTMMCl) as carboxylic acid activator in water and the corresponding azide functionalized as amine. The reaction was left stirring overnight at room temperature. After that, as all by products are either water soluble, ultrafiltration (with a membrane of $3.000 \mathrm{MWCO}$ ) or size exclusion chromatography (SEC) with Sephadex G25 columns, was done in order to purify the copolymer. A colorless amorphous solid was yielded after freeze-drying of the sample. (\% PEG(6) $\mathrm{N}_{3}$ included $16 \%$ of GAU, $\mathrm{Mn}=30400 \mathrm{Da})$<smiles>[R]NC(=O)C(CCC(=O)O[Na])NC</smiles>

PGA
1) $\mathrm{DMTMMCl} / \mathrm{ddH} \mathrm{H}_{2} \mathrm{O}$<smiles>NCCO[C@@H]([O-])C[NH3+]</smiles>

PGACOEG(6) $\mathbf{N}_{3}$

Scheme S7. Synthetic route to azide modified PGA polymers

\section{PGA-Cy5.5 Synthesis}

For Cy5.5 labeling was performed by post polymerization modification using DMTMMCl in aqueous solutions. Briefly, in a one-neck round bottom flask, PGA polymer (sodium salt form) was dissolved in $\mathrm{ddH}_{2} \mathrm{O}$. Then, the carboxylic groups were activated using DMTMMCl (0.01 eq. GAU for $1 \%$ mol GAU modification). The reaction was allowed to proceed for 10 minutes. After that time, Cy5.5 (0.01 eq. GAU for $1 \%$ 
modification) was added in $\mathrm{ddH}_{2} \mathrm{O}$. The $\mathrm{pH}$ was adjusted to 8 by adding sodium bicarbonate $1 \mathrm{M}$. The reaction was allowed to proceed for 48 hours, protected from light. For purification, the products were submitted to both Sephadex G25 and dialysis using Vivaspin ${ }^{\circledR}$ MWCO 5000. Cy5.5 contain estimation was carried out by fluorescence ( $\lambda$ ex: $595 \mathrm{~nm}, \lambda \mathrm{em}: 680 \mathrm{~nm}$ ) after the building of an appropriate calibration curve of the Cy5.5 dye in PBS 7.4 buffer. Yield: $80 \%$. Conjugation efficiency $70 \%$. Mol Cy5.5 to GAU 0.7 $\%$ mol (1 eq. per polymer chain) $3.2 \mathrm{w} \%$, MW conjugate, $23.6 \mathrm{kDa}$

Detailed probe conjugation protocol. The activatable MMP-13 probe (P-18) was conjugated through CuAAC according to the previously published method. ${ }^{[27]}$ Briefly, in a two-necked round bottom flask fitted with a stirrer bar and a stopper, 1 eq. PGAcoEG(6) $\mathrm{N}_{3}$ sodium salt was dissolved in $\mathrm{ddH}_{2} \mathrm{O}$. After that, the corresponding amount for the desired $\%$ of substitution of clickable agent (P-18) was added in dry DMF solution. Then, 5 eq. of sodium ascorbate in $\mathrm{ddH}_{2} \mathrm{O}$ were added. After that, the mixture was degassed by performing two freeze-pump-thaw cycles. Afterwards, 1 eq. of CuSO4 was added in aqueous solution to the reaction mixture. The final complete mixture, containing a proportion $\mathrm{DMF} / \mathrm{H}_{2} \mathrm{O}$ of $4: 1$, was degassed by performing another freezepump-thaw cycle and left to react at $60{ }^{\circ} \mathrm{C}$ in an oil bath for 3 days protected from the light. For purification, the solvent was completely removed under vacuum. The colored solid was re-dissolved and purified by PD10 column collecting fractions of $0.5 \mathrm{~mL}$. Two clear fractions were found corresponding with the conjugated and unconjugated probe. Additionally, dialysis using Vivaspin ${ }^{\circledR}$ MWCO 5000 was performed to those fractions belonging to the polymer-probe conjugate. The $\%$ of probe conjugated was determined by ${ }^{1} \mathrm{H}-\mathrm{NMR}$ and by spectroscopic techniques using probe fluorescence $\lambda \mathrm{ex}=595 \mathrm{~nm}$, $\lambda \mathrm{em}=680 \mathrm{~nm}$, with adequate calibration curves. PGA-P-18 Yield: $60 \%$ CE $75 \%$; mol 
probe to GAU $1.5 \%$ (2.3 eq. per polymer chain, according to fluorescence and confirmed by $\left.{ }^{1} \mathrm{H}-\mathrm{NMR}\right), 20.74 \mathrm{wt} \%$, MW= $39.0 \mathrm{kDa}$.
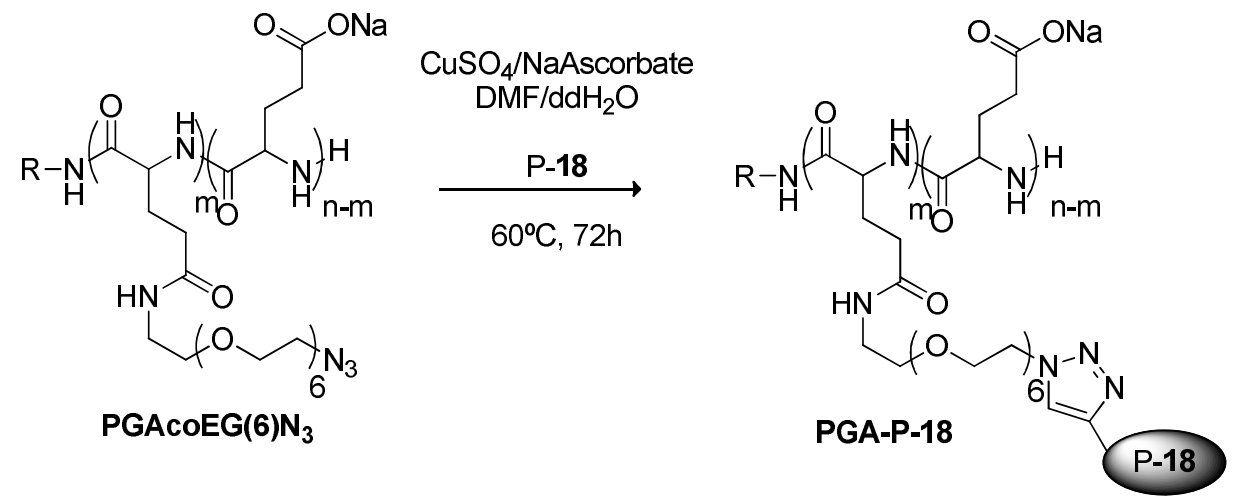

Scheme S8. Synthetic scheme for probe conjugation

\subsubsection{Synthesis of MMP-13 inhibitor A4727.}<smiles>COC(=O)c1cc(C(=O)NCc2ccc3c(c2)CCO3)ncn1</smiles><smiles>[X][M]C(=O)c1cc(C(=O)O)ncn1</smiles>

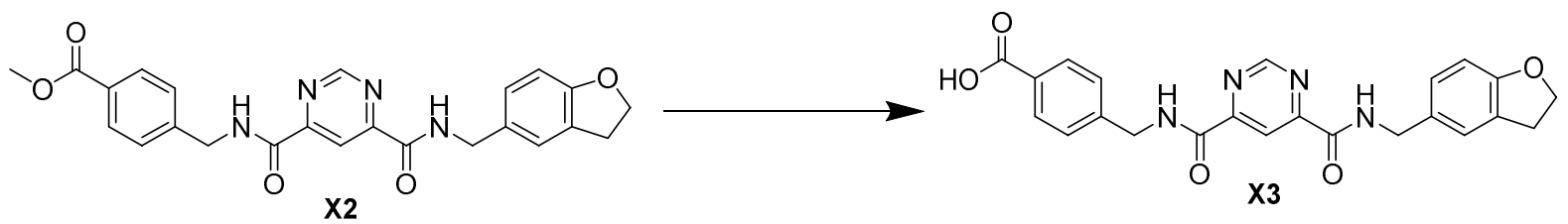<smiles>O=C(NCc1ccc(C(=O)N2CCOCC2)cc1)c1cc(C(=O)NCc2ccc3c(c2)CCO3)ncn1</smiles>

Scheme S9. Synthetic scheme for synthesis of MMP13 inhibitor A4727. 
Pyrimidine-4,6-dicarboxylic acid 4 - [(2,3-dihydro-benzofuran-5-ylmethyl) -amide] 6[4- (morpholine-4- carbonyl) -benzylamide (A4727) was synthesized in four steps.

a) Synthesis of 6-[(2,3-dihydro-benzofuran-5-ylmethyl)-carbamoyl]-pyrimidin-4$\underline{\text { carboxylic acid }(\mathbf{X 1})}$

$16.1 \mathrm{~g}(51 \mathrm{mmol})$ of 6-[(2,3-dihydro-benzofuran-5-ylmethyl)-carbamoyl]-pyrimidin-4carboxylic acid methyl ester (prepared by reaction of pyrimidine-4,6-dicarboxylic acid dimethyl ester with 5- aminomethyl-2,3-dihydrobenzofuran) were dissolved in $150 \mathrm{~mL}$ tetrahydrofuran (THF). $62 \mathrm{~mL}$ of a 1 molar aqueous $\mathrm{LiOH}$ solution was added and then the reaction mixture was stirred for 2 hours at room temperature. For workup, the reaction mixture was concentrated under reduced pressure on a rotary evaporator. The crude product was taken up in $100 \mathrm{~mL}$ of water, activated charcoal was added and filtered through Celite ${ }^{\odot}$. The solution was acidified by addition of $2 \mathrm{~N}$ aqueous $\mathrm{HCl}$ to precipitate the desired product as colorless crystals. After filtration and drying $8.3 \mathrm{~g}(27 \mathrm{mmol}, 53 \%)$ of the desired product was obtained, which could directly be further converted. $\mathrm{MS}\left(\mathrm{ES}^{+}\right)$: 300.1

b) Synthesis of 4-[(\{6-[(2,3-dihydro-benzofuran-5-ylmethyl) carbamoyl]-pyrimidine-4carbonyl\}-amino)-methyll-benzoic acid methyl ester (X2)

$4.7 \mathrm{~g}(15.7 \mathrm{mmol})$ of $\mathbf{X} \mathbf{1}$ were dissolved in $30 \mathrm{~mL}$ of dry $\mathrm{DMF}$ and cooled to $0^{\circ} \mathrm{C}$. Successively, $3.5 \mathrm{~g}$ (17.3 mmol) of 4-amino-methyl-benzoic acid methyl ester, $5.7 \mathrm{~g}$ (17.3 mmol) O-[(cyano-ethoxy-carbonyl-methylene)amino]-N,N,N',N'-tetramethyluronium tetrafluoroborate (TOTU) and $4.8 \mathrm{ml}$ of trimethylamine were added portionwise. The reaction mixture was stirred for one hour at $0^{\circ} \mathrm{C}$ and then allowed to slowly warm to room temperature, stirring was continued until reaction went to completion. For workup, the 
solvent was removed under reduced pressure and the residue was added with $100 \mathrm{~mL}$ of dichloromethane. The organic phase was then dried twice with saturated aqueous sodium bicarbonate solution and washed once with water with sodium sulfate and the solvent was removed under reduced pressure. The resulting crude product crystallized after addition of little diethyl ether as pale yellow crystals $(6.8 \mathrm{~g}(15.2 \mathrm{mmol}, 97 \%)$. The product was directly employed in the next steps, no purification was required. MS (ES $\left.{ }^{+}\right)$: 447.1 $(\mathrm{M}+\mathrm{H})^{+}$.

c) 4-[(\{6-[(2,3-dihydro-benzofuran-5-ylmethyl) carbamoyl $]$-pyrimidine-4-carbonyl $\}$ $\underline{\text { amino) - methyl] -benzoic acid (X3) }}$

$6.28 \mathrm{~g}(14 \mathrm{mmol})$ of $4-[(\{6-$ [(2,3-dihydro-benzofuran-5-ylmethyl $)$ carbamoyl $]$ pyrimidine-4- carbonyl \} -amino) - methyl] -benzoic acid methyl ester X2 were dissolved in $150 \mathrm{~mL}$ of THF. $70 \mathrm{~mL}$ of water and $16.9 \mathrm{~mL}$ of $1 \mathrm{~N}$ aqueous sodium hydroxide solution were added successively and the reaction mixture was allowed to stir for 24 hours at room temperature.

For workup, the reaction mixture was concentrated under reduced pressure to a volume of about $50 \mathrm{~mL} .100 \mathrm{~mL}$ of ice water were added and the solution was acidified using $2 \mathrm{~N}$ aqueous $\mathrm{HCl}$ solution to precipitate the product. Filtration yielded $5.4 \mathrm{~g}(12.5 \mathrm{mmol}, 89 \%)$ of $\mathbf{X} 3$ as a colorless solid. MS $\left(\mathrm{ES}^{+}\right): 433.2$

${ }^{1} \mathrm{H}-\mathrm{NMR}(400 \mathrm{MHz}, \mathrm{DMS} 0-d 6): \delta=3.13(\mathrm{t}, \mathrm{J}=8.7 \mathrm{~Hz}, 2 \mathrm{H}), 4: 43(\mathrm{~d}, \mathrm{~J}=6.1 \mathrm{~Hz}, 2 \mathrm{H}$ ), 4:48 (t, J = 8.7 Hz, $2 \mathrm{H}), 4: 59(\mathrm{~d}, \mathrm{~J}=6.3 \mathrm{~Hz}, 2 \mathrm{H}), 6.69(\mathrm{~d}, \mathrm{~J}=8.1 \mathrm{~Hz}, 1 \mathrm{H}), 7: 07(\mathrm{~m}, 1$ H), $7.22(\mathrm{~m}, 1 \mathrm{H}), 7: 44(\mathrm{~m}, 2 \mathrm{H}), 7.88(\mathrm{~m}, 1 \mathrm{H}), 7.90(\mathrm{~m}, 1 \mathrm{H}), 8: 47(\mathrm{~d}, \mathrm{~J}=1.5 \mathrm{~Hz}, 1 \mathrm{H})$, 9:46 (d, J = 1.3 Hz, $1 \mathrm{H}), 9: 56(\mathrm{t}, \mathrm{J}=6.3 \mathrm{~Hz}, 1 \mathrm{H}), 9.76(\mathrm{t}, \mathrm{J}=6.3 \mathrm{~Hz}, 1 \mathrm{H}), 12.90(\mathrm{br} \mathrm{s}$, $1 \mathrm{H})$ 
d) pyrimidine-4,6-dicarboxylic acid 4 - [(2,3-dihydro-benzofuran-5-ylmethyl) -amide]

\section{6- [4- (morpholine-4-carbonyl) -benzylamide] (A4727)}

$432 \mathrm{mg}(1 \mathrm{mmol})$ of $\mathbf{X} 3$ was dissolved in $5 \mathrm{~mL}$ of dry DMF and cooled to $0^{\circ} \mathrm{C} .96 \mu \mathrm{L}$ (1.1 mmol) of morpholine, $361 \mathrm{mg}(1.1 \mathrm{mmol})$ of 0 - [(cyano ethoxy-carbonyl-methylene) amino] $-\mathrm{N}, \mathrm{N}, \mathrm{N}$ ', N'-tetramethyluronium tetrafluoroborate (TOTU) and $155 \mu \mathrm{L}$ of trimethylamine were added successively. The reaction mixture was stirred for 1 hour at $0^{\circ} \mathrm{C}$ and 8 hours at room temperature.

For workup, the solvent was distilled off under reduced pressure and the residue dissolved in $30 \mathrm{~mL}$ of dichloromethane. The organic phase was extracted twice with saturated aqueous sodium bicarbonate solution and once with water, dried over sodium sulfate and the solvent was removed under reduced pressure. The crude product was purified by silica gel chromatography (ethyl acetate $/$ methanol $=20 / 1)$ to yield $340 \mathrm{mg}(0.68 \mathrm{mmol}, 68 \%)$ of the desired product as colorless crystals. MS (ES $\left.{ }^{+}\right): 502.27$

${ }^{1} \mathrm{H}-\mathrm{NMR}(500 \mathrm{MHz}, \mathrm{DMS} 0-d 6): \delta=2.60(\mathrm{~m}, 2 \mathrm{H}), 3.4$ - $3.6($ br m, $8 \mathrm{H}), 4: 42(\mathrm{~d}, \mathrm{~J}=$ $6.5 \mathrm{~Hz}, 2 \mathrm{H}), 4: 48(\mathrm{t}, \mathrm{J}=8.6 \mathrm{~Hz}, 2 \mathrm{H}), 4: 56(\mathrm{~d}, \mathrm{~J}=6.5 \mathrm{~Hz}, 2 \mathrm{H}), 6.68(\mathrm{~d}, \mathrm{~J}=8.3 \mathrm{~Hz}, 1$ H), 7:07 (d, J = 6.5 Hz, $1 \mathrm{H}), 7.22(\mathrm{~s}, 1 \mathrm{H}), 7.88(\mathrm{~m}, 4 \mathrm{H})$, 8:46 (m, $1 \mathrm{H})$, 9:46 (m, $1 \mathrm{H})$, 9:57 (t, J = 6.5 Hz, $1 \mathrm{H}), 9.75(\mathrm{t}, \mathrm{J} 6.5 \mathrm{~Hz}, 1 \mathrm{H})$

\subsubsection{Pharmacokinetics of $P-18$ and $P G A-P-18$}

Pharmacokinetics of P-18 and PGA-P-18 were performed in compliance with the National Animal Welfare Regulations at the CEA in Saclay. The experiments were approved by the local ethics committee for animal experimentation. C57BL/6 mice (Charles River Laboratories, L'Abrésie, France) were individually housed in polycarbonate cages in a conventional animal facility and had access ad libitum to food 
and drink. $1 \mathrm{nmol}$ of probes $1,3,4$ or 5 was injected intravenously in the tail vein $(n=3$ for each group) under anesthesia ( $2 \%$ isoflurane). At various time points $(0,1 \mathrm{~min}, 5 \mathrm{~min}$, $10 \mathrm{~min}, 15 \mathrm{~min}, 30 \mathrm{~min}, 45 \mathrm{~min}, 60 \mathrm{~min}, 90 \mathrm{~min}, 120 \mathrm{~min}, 180 \mathrm{~min}$ and $240 \mathrm{~min}$ ), blood samples (whole blood) were collected from the retro orbital sinus under anesthesia with $2 \%$ isoflurane. Animals were sacrificed by carbon dioxide asphyxiation after 4 hours and the different organs (liver, kidneys, spleen and lungs) were harvested post-mortem. Blood and organs were imaged by fluorescence reflectance using FMT 1500's planar imaging capability (Perkin Elmer, Waltham, MA). Imaging was conducted with the appropriate excitation (Ex) and emission (Em) filter sets: $\mathrm{Ex} / \mathrm{Em}=675 / 720 \mathrm{~nm}$ for probes 1,3 and 5, and $\mathrm{Ex} / \mathrm{Em}=745 / 800 \mathrm{~nm}$ for probe 4 . Blood fluorescence was converted to $\%$ injected dose per gram $(\% \mathrm{ID} / \mathrm{g})$ using a standard curve derived from serial dilutions of each probe in mouse blood. Tissue fluorescence was quantified as mean efficiency per pixel and was presented as an arbitrary unit (AU). In the case of mice injected with probes 1,3 and 4, urine was collected and analyzed by liquid chromatography (Agilent XDB C18 column) and mass spectrometry (Electrospray in a positive mode of ionization). For each probe UV detection was performed at the wavelength corresponding to the maximal absorbance of its conjugated fluorochrome.

\subsubsection{Enzyme kinetics Analysis}

Enzyme inhibition assays were carried out in $50 \mathrm{mM}$ Tris/ $\mathrm{HCl}$ buffer, $\mathrm{pH}$ 6.8, $10 \mathrm{mM}$ $\mathrm{CaCl}_{2}$, at $25{ }^{\circ} \mathrm{C}$. The assays were performed in black 96-well plates (non binding surface plates 3651; Corning Costar). Progress curves were monitored by recording the increase in fluorescence resulting from substrate cleavage (Coumarin probe: $\lambda_{\mathrm{ex}}=320 \mathrm{~nm}, \lambda_{\mathrm{em}}=$ $405 \mathrm{~nm}$; cyanine probe: $\left.\lambda_{\mathrm{ex}}=665 \mathrm{~nm}, \lambda_{\mathrm{em}}=695 \mathrm{~nm}\right)$. The conditions of a typical experiment were $100 \mu \mathrm{L}$ of buffer, 1-5 nM of MMP or cathepsins ( $\mathrm{R} \& \mathrm{R}$ systems). The 
reaction was then initiated by addition of $0.5 \mu \mathrm{M}$ of substrate. (stock solution of $100 \mu \mathrm{M}$ of substrate in DMSO). Data were collected for 1 hour. $\mathrm{k}_{\mathrm{cat}} / \mathrm{K}_{\mathrm{m}}$ values were determined from first-order full-time reaction curves $\left(\mathrm{S}<<\mathrm{K}_{\mathrm{m}} ; \mathrm{S}=0.5 \mu \mathrm{M}\right)$, fitted with the integrated Michaelis-Menten equation by nonlinear regression: $\mathrm{P}=\mathrm{S}_{0}(1-\exp (-\mathrm{kt}))$, where $\mathrm{k}=$ $\left(\mathrm{k}_{\mathrm{cat}} / \mathrm{K}_{\mathrm{m}}\right) \cdot \mathrm{E}, \mathrm{P}$ represents product concentration, and $\mathrm{S}_{0}$ is substrate concentration at $\mathrm{t}=0$, and $\mathrm{E}$ is enzyme concentration. The kinetic parameters $\mathrm{Km}$ and Kcat were estimated according to the direct linear plot method.

\subsubsection{Animal model studies}

DMM surgery was performed on 10 week old male C57BL/6J mice (Harlan Laboratories, Blackthorn, Bicester, UK) according to Glasson et al. ${ }^{[42]}$ with the exception that isofluorane $\left(2.5 \%, \mathrm{O}_{2}\right)$ was used for anesthesia, instead of the injectable anesthetics. ${ }^{[20]}$ Sham surgery consisted of the capsulotomy, but the meniscotibial ligament was not cut. All experimental protocols were performed in compliance with the UK Animals (Scientific Procedures) Act 1986 regulations for the handling and use of laboratory animals (Home Office project license PPL no:30/3129).

\subsubsection{Distribution of PGA after i.v. injection}

One hundred and fifty $\mu 1$ of $1 \mu \mathrm{M}$ Cy5.5-labelled PGA or Cy 5.5 was injected into the tail veins of naïve 10 week old male C57BL/6J mice. One day after i.v. injection, the mice were sacrificed by cervical dislocation, the heart, kidney, spleen, liver and knees were dissected and imaged in the Kodak In Vivo FX Pro (Carestream, Woodbridge, USA). The organs were excited at $630 \mathrm{~nm}$ and the emissions greater than $700 \mathrm{~nm}$ were captured for 1 min to determine the relative amount of PGA in the different organs. 


\subsubsection{Activation of probes in vivo}

Two, 4, 6, or 8 weeks after surgery, $150 \mu \mathrm{l}$ of $1 \mu \mathrm{M}$ P-18 or PGA-P-18 was delivered intravenously via the tail vein. At the time points indicated after injection, fluorescence images were obtained by exposing mice under gaseous anesthesia (2.5\% isofluorane and $\mathrm{O}_{2}$ ) in the Kodak In Vivo FX Pro (Carestream, Woodbridge, USA) to excitation at 630 $\mathrm{nm}$ and capturing the emissions greater than $700 \mathrm{~nm}$ for $1 \mathrm{~min}$. Fluorescence images were analyzed using the Carestream MI software (version 5.1, Carestream, Woodbridge, USA.). Regions of interest (ROI) were defined as a circle of 64 pixels encompassing the knee area, centered on the joint space using co-registered X-ray images as a guide. The mean fluorescence intensity (MFI) of each ROI was obtained, without any background intensity subtraction. X-ray images were taken at the same time for co-registration of signal to the knee joint, by exposure to X-rays for $20 \mathrm{sec}$.

\subsubsection{Real time readout of the effect of MMP-13 inhibitor in the DMM} surgery model of $O A$

Mice were subjected to the DMM surgery. Two weeks after surgery, they were divided into 3 treatment groups. The first group received daily the optimal dose of MMP-13 inhibitor A4727 $30 \mathrm{mg} / \mathrm{kg}$, the second group received $0.03 \mathrm{mg} / \mathrm{kg} \mathrm{A} 4727$, and the third group received the same volume similarly. To follow the efficacy of the inhibitor, fluorescence and X-ray images of the mice were obtained two days after i.v. injection of $150 \mu \mathrm{l}$ of $1 \mu \mathrm{M}$ PGA-P-18 at 6, 8, 10 and 12 weeks post-DMM surgery. 


\subsubsection{Histology and scoring of cartilage damage}

At 12 week time point after DMM surgery, mice were sacrificed by cervical dislocation and the knee joints were dissected and fixed in formalin $(2 \%(\mathrm{v} / \mathrm{v}))$ overnight. The knee joints were isolated, decalcified and embedded in paraffin. Serial section $5 \mathrm{~mm}$ in thickness were cut. Every $20^{\text {th }}$ section was stained with Safranin O for scoring of cartilage damage. Scoring was performed by two different observers, blinded to the treatment groups, in accordance with the guidelines laid out in the OARSI histopathology initiative for small animals on a scale from 1 to $6{ }^{[4]}$ All four quadrants of the sections (medial tibial plateau, lateral tibial plateau, medial femoral condyle, lateral femoral condyle) were scored individually and the scores from all stained sections were added to obtain the summed score 


\section{Supporting Figures}

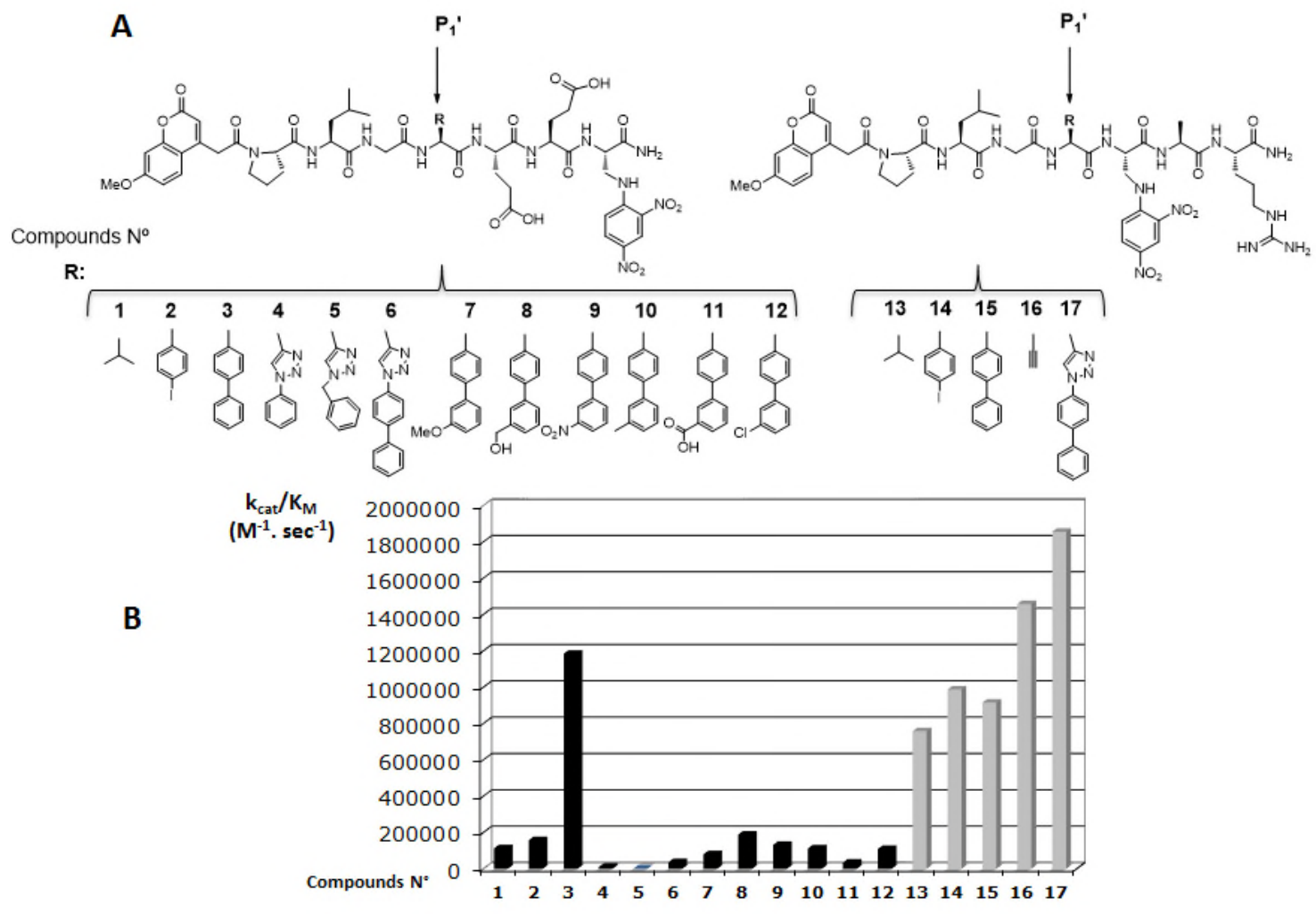

Figure S1. A) chemical structure of the probes, modifications have been introduced in the P1' position of the probe using the different reported side chains ( $\mathrm{R}$ groups); $\mathrm{B}$ ) catalytic efficiency $\left(\mathrm{k}_{\mathrm{cat}} / \mathrm{K}_{\mathrm{M}}\right)$ of recombinant MMP-13 in cleaving probes at $\mathrm{pH} 6.8$ and $25^{\circ} \mathrm{C}$ (HEPES $25 \mathrm{mM}, 1 \mathrm{mM} \mathrm{CaCl} 2,0.2 \mathrm{M} \mathrm{NaCl}$; probe concentration $0.5 \mathrm{mM}$ :MMP$13,1 \mathrm{nM})$.

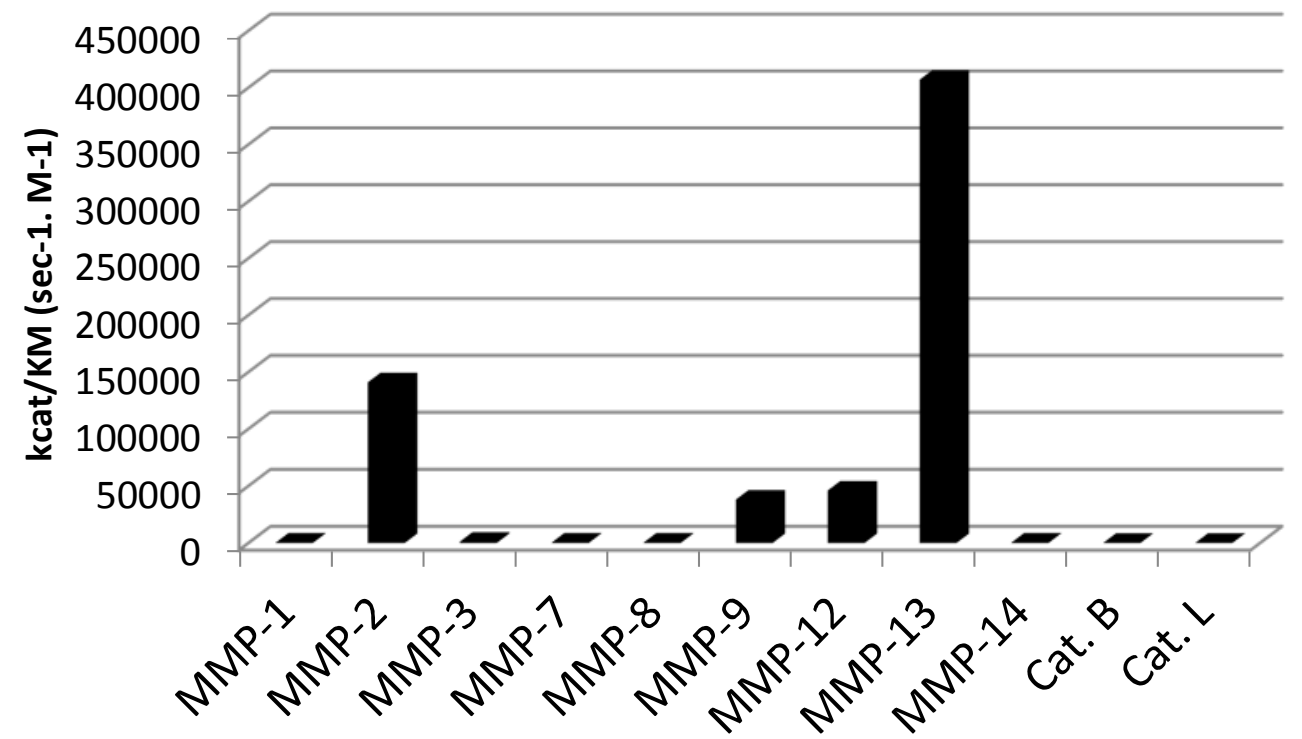


Figure S2. Catalytic efficiency $\left(\mathrm{k}_{\mathrm{cat}} / \mathrm{K}_{\mathrm{M}}\right)$ of recombinant MMPs and cathepsins in cleaving the probe $\mathrm{P}-18$ at $\mathrm{pH} 6.8$ and $25^{\circ} \mathrm{C}\left(25 \mathrm{mM}\right.$ Hepes, $1 \mathrm{mM} \mathrm{CaCl}_{2}, 0.2 \mathrm{M} \mathrm{NaCl}$; probe concentration $0.5 \mathrm{mM}$; enzymes, $1 \mathrm{nM}$ ).

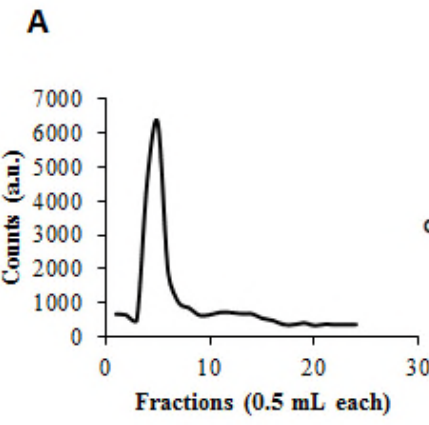

B

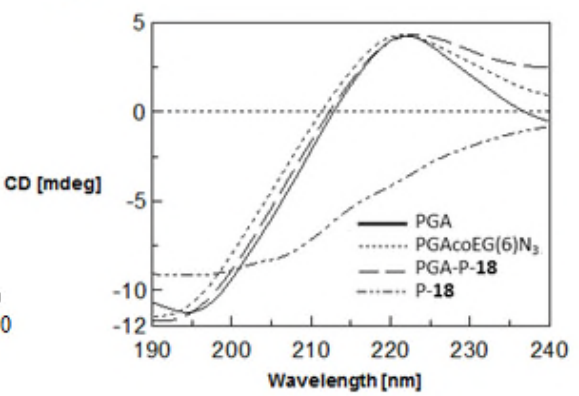

C
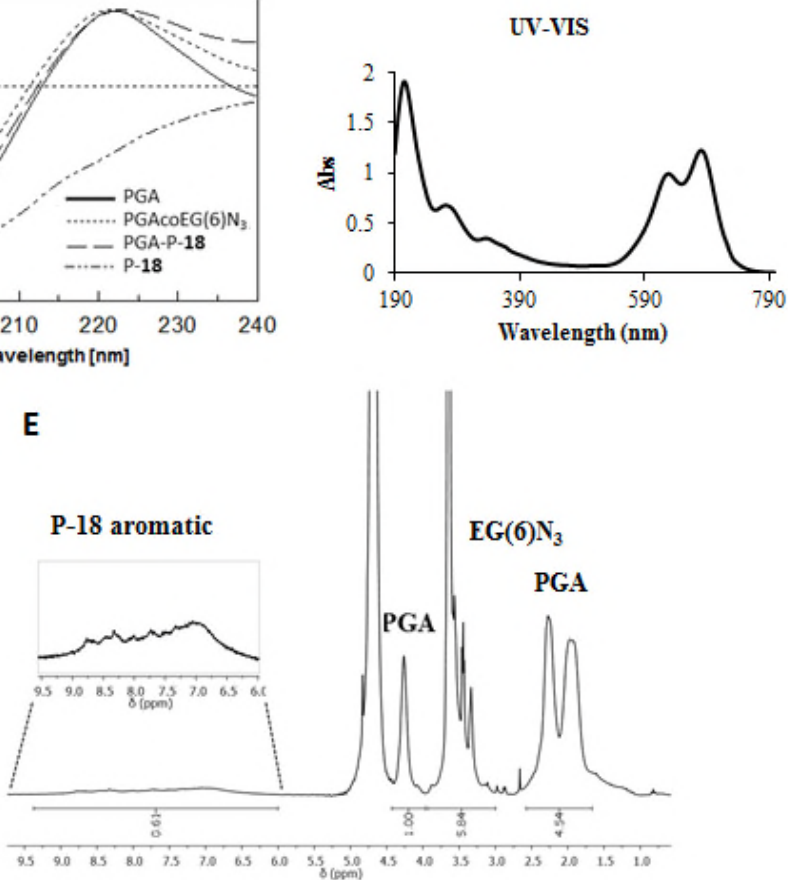

Figure S3. Physico-chemical characterization of PGA-P-18. A) Fluorescence quantification of PGA-P-18 eluted fractions obtained by SEC purification. B) Circular Dichroism (CD) of PGA derivatives including PGA, PGAcoEG(6) $\mathrm{N}_{3}$ and the polymeric probe PGA-P-18 in comparison with the free probe. Random coil conformation is retained in PGA derivatives after peptidic probe conjugation. C) UV-VIS spectrum of PGA-P-18 in water. D) Z-potential of PGA-P-18 in water at $0.1 \mathrm{mg} \cdot \mathrm{mL}^{-1}$ (z-potential:$23.00 \pm 4.4 \mathrm{mV}$ ). E) ${ }^{1} \mathrm{H}-\mathrm{NMR}$ in $\mathrm{D}_{2} \mathrm{O}$ of PGA-P-18 showing assignations. 


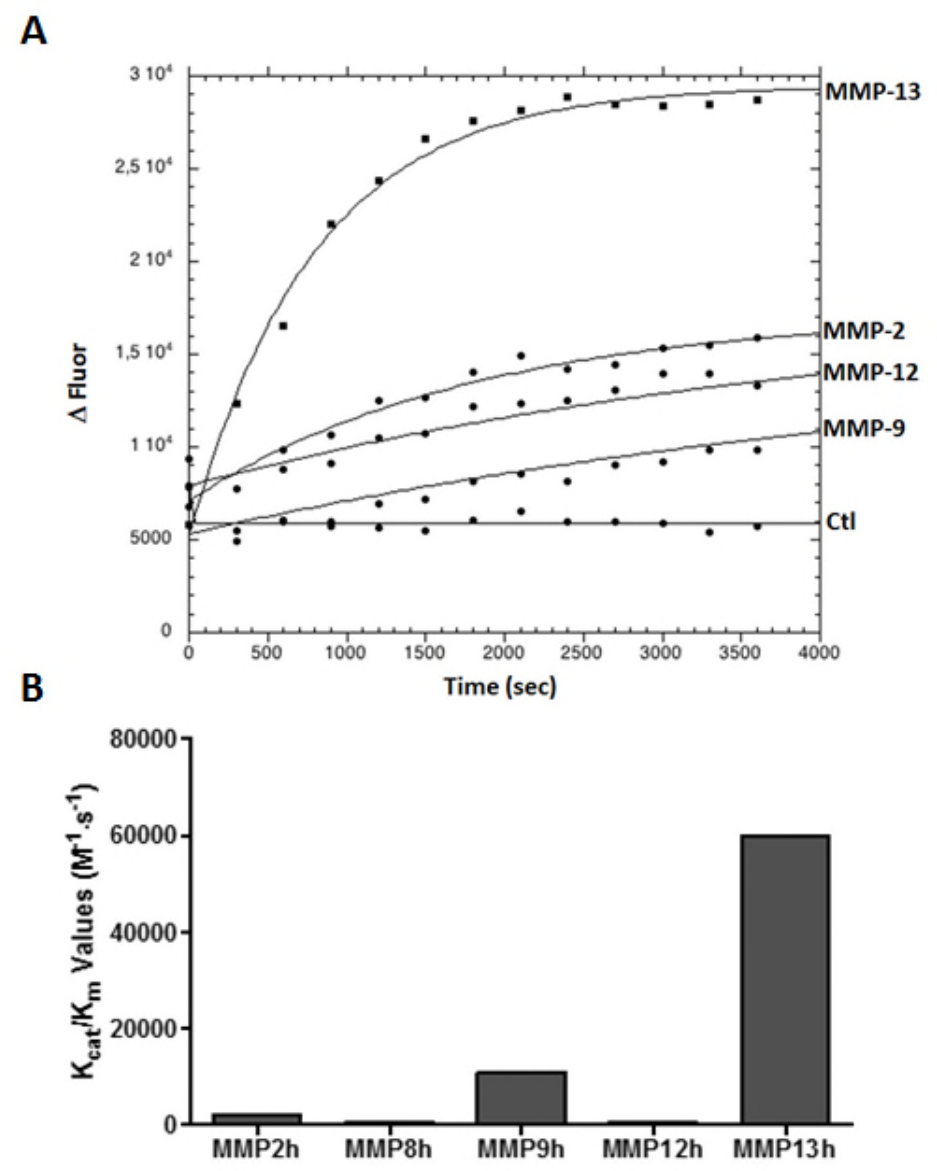

Figure S4. A) Progress curves of degradation of the PGA-18 probe by MMPs; in diluted mouse plasma (1/10 dilution), in the buffer ( $\mathrm{pH} 6.825^{\circ} \mathrm{C}$, Hepes $25 \mathrm{mM}, 1 \mathrm{mM} \mathrm{CaCl}_{2}$, $0.2 \mathrm{M} \mathrm{NaCl}$; probe concentration $0.5 \mathrm{mM}$, enzymes, $1 \mathrm{nM}$ ). In control experiment (Ctl), no MMP was added, showing the probe stability under these conditions. B) $\mathrm{K}_{\mathrm{cat}} / \mathrm{K}_{\mathrm{m}}$ values obtained from the enzymatic curves in diluted mouse plasma. 
A

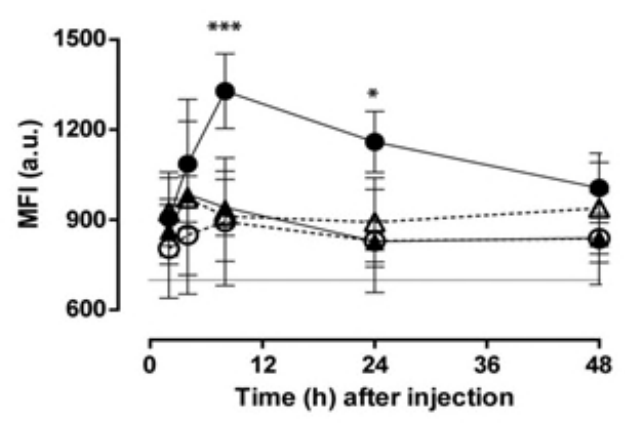

B
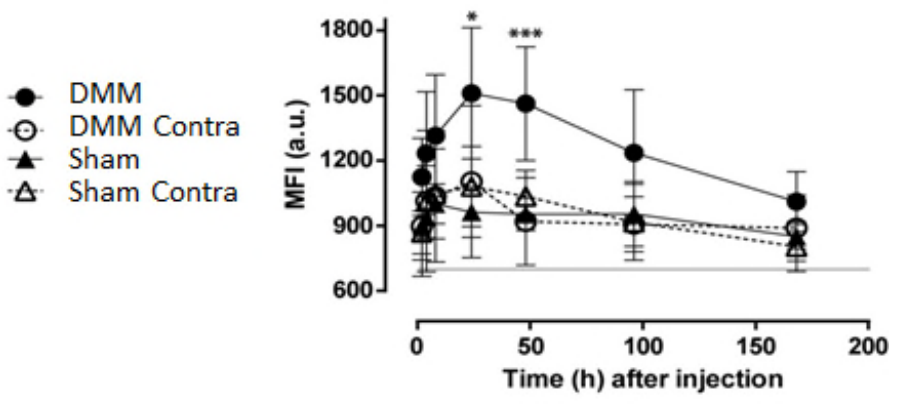

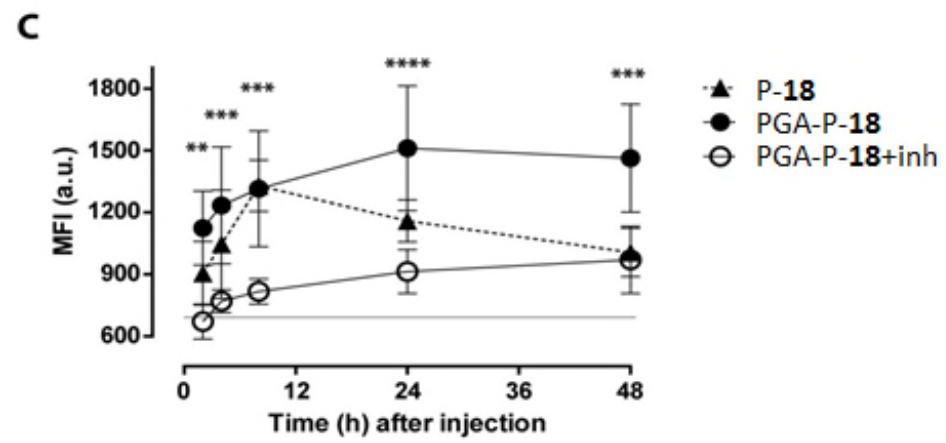

Figure S5. Activation of P-18 and PGA-P-18 in vivo at 8 weeks following DMM surgery. (A) Activation of P-18 following i.v. injection; (B) Activation of PGA-P-18 following i.v. injection; (C) Inhibition of activation of PGA-P-18 in the presence of the MMP-13 inhibitor. Data are mean $\pm \mathrm{SD}, \mathrm{n}=5 . * \mathrm{p}<0.05, * \mathrm{p}<0.01$, *** $\mathrm{p}<0.005$, **** $\mathrm{p}<$ 0.001 between. DMM and other groups (A, B), and between P-18 and PGA-P-18 (C) after two-way ANOVA with Bonferroni post tests.

\section{Supporting Tables}

Table S1. HPLC and mass-spectrometry data.

\begin{tabular}{lccccc}
\hline $\begin{array}{l}\text { Compound } \\
\mathbf{N}^{\circ}\end{array}$ & $\begin{array}{l}\text { HPLC, } \\
(\mathbf{m i n})\end{array}$ & retention time & $\begin{array}{l}\text { MS, } \\
\text { calc }\end{array}$ & $\begin{array}{l}\mathbf{M S}, \mathbf{m} / \mathbf{z}, \\
{[\mathbf{M}+\mathbf{H}]+}\end{array}$ & found \\
\hline $\mathbf{1}$ & 18.5 & 1122.48 & 1199.5 \\
$\mathbf{2}$ & 19.7 & 1283.33 & 1284.3 \\
$\mathbf{3}$ & 20.0 & 1233.46 & 1234.5 \\
$\mathbf{4}$ & 18.9 & 1224.44 & 1225.4 \\
$\mathbf{5}$ & 19.0 & 1238.46 & 1239.5 \\
$\mathbf{6}$ & 20.1 & 1300.47 & 1301.4 \\
$\mathbf{7}$ & 20.0 & 1277.47 & 1278.5 \\
$\mathbf{8}$ & 19.1 & 1263.47 & 1264.2
\end{tabular}




\begin{tabular}{llll}
$\mathbf{9}$ & 20.1 & 1278.44 & 1279.6 \\
$\mathbf{1 0}$ & 20.5 & 1247.47 & 1248.4 \\
$\mathbf{1 1}$ & 19.1 & 1277.45 & 1278.3 \\
$\mathbf{1 2}$ & 20.7 & 1267.42 & 1268.3 \\
$\mathbf{1 3}$ & 18.6 & 1091.32 & 1092.1 \\
$\mathbf{1 4}$ & 19.8 & 1252.37 & 1253.3 \\
$\mathbf{1 5}$ & 20.2 & 1202.51 & 1203.5 \\
$\mathbf{1 6}$ & 18.5 & 1074.45 & 1075.4 \\
$\mathbf{1 7}$ & 20.2 & 1269.53 & 1270.2 \\
$\mathbf{1 8}$ & 12.9 & 1240.79 & 1240.9 \\
\hline
\end{tabular}

Table S2. Characterization of PGA precursors and conjugate.

\begin{tabular}{llll} 
Compound & Mn $(\mathbf{k D a})$ & Đ & D $(\mathbf{n m})$ \\
\hline PGA & 19.6 & 1.2 & 13.5 \\
PGAcoEG(6)N3 & 30.4 & 1.2 & 7.3 \\
PGA-P-18 & 39.0 & 1.2 & 7.9 \\
\hline
\end{tabular}

* Molar mass averages of the number (Mn) in $\mathrm{kDa}$, and (Polydispersity) $Đ$ as obtained by gel permeation chromatography (GPC) and the Hydrodynamic diameter (D) as obtained by the diffusion coefficient by Diffusion-ordered spectroscopy nuclear magnetic resonance (DOSY-NMR) 